TRANSACTIONS OF THE

AMERICAN MATHEMATICAL SOCIETY

Volume 350, Number 4, April 1998, Pages 1359-1378

S 0002-9947(98)02136-9

\title{
COVERS OF ALGEBRAIC VARIETIES III. THE DISCRIMINANT OF A COVER OF DEGREE 4 AND THE TRIGONAL CONSTRUCTION
}

\author{
G. CASNATI
}

\begin{abstract}
For each Gorenstein cover $\varrho: X \rightarrow Y$ of degree 4 we define a scheme $\Delta(X)$ and a generically finite map $\Delta(\varrho): \Delta(X) \rightarrow Y$ of degree 3 called the discriminant of $\varrho$. Using this construction we deal with smooth degree 4 covers $\varrho: X \rightarrow \mathbb{P}_{\mathbb{C}}^{n}$ with $n \geq 5$. Moreover we also generalize the trigonal construction of S. Recillas.
\end{abstract}

\section{INTRODUCTION AND NOTATIONS}

In $[\mathrm{C}-\mathrm{E}]$ a general structure theorem for Gorenstein covers of degree $d$ has been proved. Such a result has been used for characterizing covers of degree 3,4 and 5 (see $[\mathrm{C}-\mathrm{E}]$ and $[\mathrm{Cs}]$ respectively) of algebraic varieties. More precisely, in section 4 (resp. 3) of $[\mathrm{C}-\mathrm{E}]$ it is proved that the total space $X$ of each Gorenstein cover $\varrho: X \rightarrow Y$ of degree 4 (resp. 3) is obtained inside a suitable projective bundle $\pi: \mathbb{P} \rightarrow Y$ of rank 2 (resp. 1) as the base locus of a pencil of relative conics (resp. the zero locus of a relative cubic form in two variables), and $\varrho=\pi_{\mid X}$.

For each pencil of conics $\mathfrak{b}$ in $\mathbb{P}_{k}^{2}$ spanned by two quadratic forms $a:=x^{t} A x$ and $b:=x^{t} B x$ ( $A$ and $B$ being symmetric $3 \times 3$ matrices $)$ the discriminant $\Delta(\mathfrak{b})$ is the cubic (possibly zero) polynomial $\Delta(\mathfrak{b})=\operatorname{det}(s A+t B)$. The roots of $\Delta(\mathfrak{b})$ correspond to the degenerate conics of $\mathfrak{b}$.

In this paper we investigate the geometry of the Galois-theoretic relationship between the general equations of degree 4 and 3 . We are thus able to generalize in arbitrary dimension the trigonal construction due to S. Recillas for covers of $\mathbb{P}_{k}^{1}$ (see [Re]). We do this globalizing the description above (exploiting an idea due to T. Ekedahl: see $[\mathrm{Ek}])$.

In section 4 we define for each Gorenstein cover $\varrho: X \rightarrow Y$ of degree 4 its discriminant $\Delta(\varrho): \Delta(X) \rightarrow Y$, getting the following result.

Theorem 1.1. Let $Y$ be an integral scheme defined over an algebraically closed field $k$ of characteristic $p \neq 2$. To each Gorenstein cover $\varrho: X \rightarrow Y$ of degree 4 one can associate its discriminant $\Delta(\varrho): \Delta(X) \rightarrow Y$, which is generically finite of degree 3 . The points $y \in Y$ over which $\Delta(\varrho)$ does not have finite fibres are exactly

Received by the editors December 1, 1995.

1991 Mathematics Subject Classification. Primary 14E20, 14E22.

Key words and phrases. Cover, Gorenstein, discriminant.

This work was done in the framework of the AGE project, H.C.M. contract ERBCHRXCT 940557. 
the points such that

$$
\varrho^{-1}(y) \cong \operatorname{spec}\left(\frac{k(y)[u, v]}{\left(u^{2}, v^{2}\right)}\right),
$$

and in this case $\Delta(\varrho)^{-1}(y) \cong \mathbb{P}_{k(y)}^{1}$. If there are not such $y^{\prime}$, then $\Delta(\varrho)$ is a Gorenstein cover of degree 3 .

Moreover we also study the reducedness, connectedness and smoothness of the discriminant cover, giving many examples exhibiting various possible behaviours.

In section 5 we determine the class of $\operatorname{Sing}(\Delta(X))$ under the hypotheses of genericity of $\varrho: X \rightarrow Y$.

Section 6 is devoted to the generalization of the above mentioned trigonal construction to covers of arbitrary smooth schemes $Y$ satisfying some mild technical conditions (see Theorem 6.3).

In section 7 we deal with covers of $\mathbb{P}_{\mathbb{C}}^{n}$.

Theorem 1.2. If $X$ is integral and smooth, $n \geq 5$, and $\varrho: X \rightarrow \mathbb{P}_{\mathbb{C}}^{n}$ is a cover of degree 4 such that $\varrho_{*} \mathcal{O}_{Y} / \mathcal{O}_{\mathbb{P}_{\mathbb{C}}^{n}}$ is uniform, then $\varrho_{i}^{*}: H^{i}\left(\mathbb{P}_{\mathbb{C}}^{n}, \mathbb{C}\right) \rightarrow H^{i}(X, \mathbb{C})$ is an isomorphism for $i \leq 0 \leq n-1$ and a monomorphism (but not an isomorphism) for $i=n$.

Taking into account Fujita's description of covers $\varrho: X \rightarrow Y$ of degree 3 with both $X$ and $Y$ smooth (see [Fj]), we are also able to give a qualitative study of the locus of points of total ramification of a cover of degree 4 .

Theorem 1.3. If $\varrho: X \rightarrow \mathbb{P}_{\mathbb{C}}^{n}$ is a cover of degree 4 with $X$ smooth and $n \geq 5$, then $X$ is a quadrisecant of an ample line bundle if and only if for each $y \in \mathbb{P}_{\mathbb{C}}^{n}$ there is an embedding $X_{y}:=\varrho^{-1}(y) \hookrightarrow \mathbb{A}_{\mathbb{C}}^{1}$. More precisely, either $X$ is a quadrisecant of an ample line bundle, or there is a point $y \in \mathbb{P}_{\mathbb{C}}^{n}$ such that the fibre of $\varrho$ over $y$ is isomorphic to

$$
\varrho^{-1}(y) \cong \operatorname{spec}\left(\frac{\mathbb{C}[u, v]}{\left(u^{2}, v^{2}\right)}\right) .
$$

In this case $y$ has at least multiplicity 4 in the branch locus of $\varrho$.

Now we recall some definitions, notations and results which will be used in what follows.

A local ring $R$ is Cohen-Macaulay if $\operatorname{dim}(R)=\operatorname{depth}(R)$. A Cohen-Macaulay $\operatorname{ring} R$ is called Gorenstein if its injective dimension $i d_{R}(R)$ is finite. An arbitrary ring $R$ is called Cohen-Macaulay (resp. Gorenstein) if $R_{\mathfrak{M}}$ is Cohen-Macaulay (resp. Gorenstein) for every maximal ideal $\mathfrak{M} \subseteq R$.

In this paper all schemes are assumed to be noetherian. All schemes over a field $k$ of characteristic $p$ are assumed to be separated and of finite type over $k$, and we always take $p \neq 2$.

A scheme $X$ is Cohen-Macaulay (resp. Gorenstein) if for each point $x \in X$ the local ring $\mathcal{O}_{X, x}$ of $X$ in $x$ is Cohen-Macaulay (resp. Gorenstein). If $X$ is defined over $k$, then it is Gorenstein if and only if it is Cohen-Macaulay and its dualizing sheaf $\omega_{X \mid k}$ is invertible.

Let $Y$ be a scheme. A flat morphism $\varrho: X \rightarrow Y$ is said to be Gorenstein of relative dimension $r$ if for each $y \in Y$ the scheme-theoretic fibre $X_{y}:=\varrho^{-1}(y)$ is a Gorenstein scheme over $k(y)$ of dimension $r$. In particular, the relative dualizing sheaf is defined and invertible. 
Let $Y$ be an scheme. A morphism $\varrho: X \rightarrow Y$ is called a cover of degree $d$ if $\varrho_{*} \mathcal{O}_{X}$ is a locally free $\mathcal{O}_{Y}$-sheaf of rank $d: \varrho$ is a cover if and only if it is flat and finite. If $Y$ is smooth and $X$ is Cohen-Macaulay, then every finite surjective morphism is a cover.

There exists an exact sequence of the form $0 \rightarrow \mathcal{O}_{Y} \rightarrow \varrho_{*} \mathcal{O}_{X} \rightarrow \check{\mathcal{E}} \rightarrow 0$, where $\check{\mathcal{E}}$ is a locally free $\mathcal{O}_{Y}$-sheaf of rank $d-1$ called the Tschirnhausen module of $\varrho$. If the characteristic $p$ of $k$ does not divide $d$, then the above sequence splits and $\varrho_{*} \mathcal{O}_{X} \cong \mathcal{O}_{Y} \oplus \check{\mathcal{E}}$ (generalize the proof of lemma 2.2 in [Mi]). If the cover $\varrho: X \rightarrow Y$ is Gorenstein, then $\left(\varrho_{*} \mathcal{O}_{X}\right)^{-} \cong \varrho_{*} \omega_{X \mid Y}$ (see [Ha], exercise III 6.10), and hence $\varrho_{*} \omega_{X \mid Y} \cong \mathcal{O}_{Y} \oplus \mathcal{E}$.

If $\mathcal{E}$ is a locally free $\mathcal{O}_{Y}$-sheaf of rank $d+1$, we denote by $\pi: \mathbb{P}(\mathcal{E}) \rightarrow Y$ the corresponding projective bundle, i.e. $\mathbb{P}(\mathcal{E}):=\operatorname{Proj}(\mathcal{S} \mathcal{E})(\mathcal{S} \mathcal{E}$ denotes the symmetric algebra of $\mathcal{E}, \mathcal{S}^{n} \mathcal{E}$ its component of degree $n$ ), and $\pi$ is induced by the natural monomorphism $\mathcal{O}_{Y} \longmapsto \mathcal{S E}$ (see [Ha]).

If $\eta \in H^{0}(Y, \mathcal{F})$ is a section of a locally free $\mathcal{O}_{Y}$-sheaf $\mathcal{F}$, we denote by $D_{0}(\eta)$ its zero-locus.

I would like to express my thanks to F. Catanese for a number of helpful discussions and interesting suggestions.

\section{Covers of Degree 3 And 4}

For the following results we refer to sections 3 and 4 of [C-E]. Let $X$ and $Y$ be schemes, $Y$ integral, and let $\varrho: X \rightarrow Y$ be a Gorenstein cover of degree $d=3$ or $d=4$. There exists a natural factorization $\varrho=\pi \circ i$, where $\pi: \mathbb{P} \rightarrow Y$ is the natural projection, $\mathbb{P}:=\mathbb{P}(\mathcal{E}), \check{\mathcal{E}}$ being the Tschirnhausen module of $\varrho$ and $i: X \hookrightarrow \mathbb{P}$ is a closed embedding. Moreover, $\omega_{X \mid Y} \cong \mathcal{O}_{\mathbb{P}}(1)_{\mid X}$, and for each $y \in Y$ the fibre $X_{y}:=\varrho^{-1}(y)$ is a complete intersection in $\mathbb{P}_{y}:=\pi^{-1}(y)$. If $d=3$ then $X_{y} \subseteq \mathbb{P}_{y} \cong \mathbb{P}_{k(y)}^{1}$ is the zero locus of a single cubic polynomial, whereas if $d=4$ then $X_{y} \subseteq \mathbb{P}_{y} \cong \mathbb{P}_{k(y)}^{2}$ is the base locus of a pencil of conics without fixed components.

If $d=3$ there is a unique exact sequence

$$
0 \rightarrow \pi^{*} \operatorname{det} \mathcal{E}(-3) \stackrel{\delta}{\rightarrow} \mathcal{O}_{\mathbb{P}} \rightarrow \mathcal{O}_{X} \rightarrow 0 .
$$

If $d=4$ there is a unique locally free $\mathcal{O}_{Y}$-sheaf $\mathcal{F}$ of $\operatorname{rank} 2$ such that $\operatorname{det} \mathcal{F} \cong \operatorname{det} \mathcal{E}$ and an exact sequence

$$
0 \rightarrow \pi^{*} \operatorname{det} \mathcal{E}(-4) \rightarrow \pi^{*} \mathcal{F}(-2) \stackrel{\delta}{\rightarrow} \mathcal{O}_{\mathbb{P}} \rightarrow \mathcal{O}_{X} \rightarrow 0 .
$$

In particular, in both the above cases, $X=D_{0}(\delta)$. Using the natural isomorphisms

$$
\begin{aligned}
\Phi_{3}: H^{0}\left(Y, \mathcal{S}^{3} \mathcal{E} \otimes \operatorname{det} \mathcal{E}^{-1}\right) & \stackrel{\sim}{\longrightarrow} H^{0}\left(\mathbb{P},\left(\pi^{*} \operatorname{det} \mathcal{E}^{-1}\right)(3)\right), \\
\Phi_{4}: H^{0}\left(Y, \check{\mathcal{F}} \otimes \mathcal{S}^{2} \mathcal{E}\right) & \stackrel{\sim}{\longrightarrow} H^{0}\left(\mathbb{P},\left(\pi^{*} \check{\mathcal{F}}\right)(2)\right),
\end{aligned}
$$

one gets sections $\eta:=\Phi_{3}^{-1}(\delta)$ or $\eta:=\Phi_{4}^{-1}(\delta)$. Note that $\operatorname{dim}\left(D_{0}\left(\delta_{y}\right)\right)=0$ for each point $y \in Y$. In this case we say briefly that $\eta$ has the right codimension at $y \in Y$.

Theorem 2.3. Let $Y$ be an integral scheme. Any Gorenstein cover $\varrho: X \rightarrow Y$ of degree 3 such that $\check{\mathcal{E}} \cong$ coker $\varrho^{\#}$ determines, up to scalars,

$$
\eta \in H^{0}\left(Y, \mathcal{S}^{3} \mathcal{E} \otimes \operatorname{det} \mathcal{E}^{-1}\right)
$$

having the right codimension at every $y \in Y$. 
Conversely, given $\eta \in H^{0}\left(Y, \mathcal{S}^{3} \mathcal{E} \otimes \operatorname{det} \mathcal{E}^{-1}\right)$ having the right codimension at every $y \in Y$, let $X:=D_{0}\left(\Phi_{3}(\eta)\right) \subseteq \mathbb{P}$. Then the restriction $\varrho$ of the canonical map $\pi: \mathbb{P} \rightarrow Y$ to $X$ is a Gorenstein cover of degree 3 such that $\check{\mathcal{E}} \cong$ coker $\varrho^{\#}$.

Proof. See [C-E], theorem 3.4.

Theorem 2.4. Let $Y$ be an integral scheme. As explained above, any Gorenstein cover $\varrho: X \rightarrow Y$ of degree 4 such that $\check{\mathcal{E}} \cong$ coker $\varrho^{\#}$ determines a locally free $\mathcal{O}_{Y^{-}}$ sheaf $\mathcal{F}$ of rank 2 with $\operatorname{det} \mathcal{F} \cong \operatorname{det} \mathcal{E}$ and, up to scalars, $\eta \in H^{0}\left(Y, \check{\mathcal{F}} \otimes \mathcal{S}^{2} \mathcal{E}\right)$ having the right codimension at every $y \in Y$.

Conversely, given a locally free $\mathcal{O}_{Y}$-sheaf $\mathcal{F}$ of rank 2 with $\operatorname{det} \mathcal{F} \cong \operatorname{det} \mathcal{E}$ and $\eta \in H^{0}\left(Y, \mathcal{\mathcal { F }} \otimes \mathcal{S}^{2} \mathcal{E}\right)$ having the right codimension at every $y \in Y$, let $X:=$ $D_{0}\left(\Phi_{4}(\eta)\right) \subseteq \mathbb{P}$. Then the restriction $\varrho$ of the canonical map $\pi: \mathbb{P} \rightarrow Y$ to $X$ is a Gorenstein cover of degree 4 such that $\check{\mathcal{E}} \cong \operatorname{coker} \varrho^{\#}$ and $\mathcal{F} \cong \operatorname{ker}\left(\mathcal{S}^{2} \mathcal{E} \rightarrow \varrho_{*} \omega_{X \mid Y}^{2}\right)$.

Proof. See [C-E], theorem 4.4.

It is helpful to know when sections having the right codimension at each point exist.

Theorem 2.5. Let $Y$ be a projective, smooth, connected scheme over a field $k, \mathcal{E}$ and $\mathcal{F}$ be locally free $\mathcal{O}_{Y}$-sheaves of ranks 3 and 2 respectively, $\operatorname{det} \mathcal{E} \cong \operatorname{det} \mathcal{F}$, and define $\mathcal{H}:=\mathcal{S}^{2} \mathcal{E} \otimes \check{\mathcal{F}}$. The sets

$$
\begin{gathered}
H_{r c}:=\left\{\eta \in H^{0}(Y, \mathcal{H}) \mid \eta \text { has the right codimension at every } y \in Y\right\}, \\
H_{s}:=\left\{\eta \in H_{r c} \mid D_{0}\left(\Phi_{4}(\eta)\right) \subseteq \mathbb{P} \text { is smooth }\right\}
\end{gathered}
$$

are open (but possibly empty). If $k=\mathbb{C}$, $\operatorname{dim}(Y) \leq 3$ and $\mathcal{H}$ is globally generated, then $H_{s} \neq \emptyset . D_{0}\left(\Phi_{4}(\eta)\right)$ is connected for any $\eta \in H_{r c}$ if and only if $h^{0}(Y, \check{\mathcal{E}})=0$.

Proof. See [C-E], theorem 4.5. See also [C-E], theorem 3.6, for the degree 3 case.

\section{The BRANCh LOCUS OF A COVER OF DEGREe 4}

Let $\varrho: X \rightarrow Y$ be a Gorenstein cover of degree 4 with invariants $\mathcal{E}, \mathcal{F}$ and defined by a section $\eta \in H^{0}\left(Y, \breve{\mathcal{F}} \otimes \mathcal{S}^{2} \mathcal{E}\right)$. From now on we will denote by $\mathbb{P}:=\mathbb{P}(\mathcal{E}) \stackrel{\pi}{\rightarrow} Y$, $\overline{\mathbb{P}}:=\mathbb{P}(\mathcal{F}) \stackrel{\bar{\pi}}{\rightarrow} Y$ the canonical projections.

According to the results of section 2, for each $y \in Y$ we can define the Segre number of $X_{y}$, denoted by $S\left(X_{y}\right)$, as the Segre number of the pencil of conics of $\mathbb{P}_{y} \cong \mathbb{P}_{k(y)}^{2}$ cutting out the scheme $X_{y}$ (see [Wa]). We have the Table 3.1 (see [Wa], table 0$)$.

Definition 3.2. Let $\varrho: X \rightarrow Y$ be a Gorenstein cover of degree 4 and $y \in Y$. We say that $\varrho$ is planar (resp. even) over $y$ if $S\left(X_{y}\right)=[1,1 ; ; 1]\left(\right.$ resp. $S\left(X_{y}\right)=$ $[(1,1), 1],[(2,1)])$ and we define the two sets $R_{\text {planar }}(\varrho):=\{x \in X \mid \varrho$ is planar at $\varrho(x)\}\left(\right.$ resp. $R_{\text {even }}(\varrho):=\{x \in X \mid \varrho$ is even at $\varrho(x)\}$.

Let $\mathcal{G}:=\bar{\pi}^{*} \mathcal{E}$. Since $\mathcal{F} \cong \check{\mathcal{F}} \otimes \operatorname{det} \mathcal{F}$ and $\operatorname{det} \mathcal{F} \cong \operatorname{det} \mathcal{E}$, there is an isomorphism $\Psi: H^{0}\left(Y, \check{\mathcal{F}} \otimes \mathcal{S}^{2} \mathcal{E}\right) \rightarrow H^{0}\left(\overline{\mathbb{P}}, \mathcal{S}^{2} \mathcal{G} \otimes \operatorname{det} \mathcal{G}^{-1}(1)\right)$. In the following we will identify $\vartheta:=\Psi(\eta)$ with the corresponding symmetric map $\vartheta: \check{\mathcal{G}}(-1) \rightarrow \mathcal{G} \otimes \operatorname{det} \mathcal{G}^{-1}$. Its adjoint $\operatorname{adj}(\vartheta): \mathcal{G} \rightarrow \check{\mathcal{G}}(2)$ is symmetric too. By applying $\bar{\pi}_{*}$ we get

$$
\bar{\eta} \in \operatorname{Hom}_{\mathcal{O}_{Y}}\left(\mathcal{S}^{2} \mathcal{E}, \mathcal{S}^{2} \mathcal{F}\right) \cong \operatorname{Hom}_{\mathcal{O}_{\overline{\mathbb{P}}}}\left(\mathcal{S}^{2} \mathcal{G}, \mathcal{O}_{\overline{\mathbb{P}}}(2)\right) .
$$


TABLE 3.1

$\begin{array}{rll} & S\left(X_{y}\right) & X_{y} \subseteq \mathbb{P}_{k(y)}^{2} \\ \text { i) } & {[1,1,1]} & V_{+}\left(u^{2}+w^{2}, v^{2}+w^{2}\right) \\ \text { ii) } & {[2,1]} & V_{+}\left(u v, v^{2}+w^{2}\right) \\ \text { iii) } & {[(1,1), 1]} & V_{+}\left(u^{2}+v^{2}, w^{2}\right) \\ \text { iv) } & {[3]} & V_{+}\left(v^{2}+2 u w, v w\right) \\ \text { v) } & {[(2,1)]} & V_{+}\left(w^{2}+2 u v, v^{2}\right) \\ \text { vi) } & {[1,1 ; ; 1]} & V_{+}\left(u^{2}, v^{2}\right)\end{array}$

Lemma 3.3. $\bar{\eta}$ has rank 1 (resp. 2) at $y$ if and only if $\varrho$ is planar (resp. even) over $y$.

Proof. We treat only case i) of Table 3.1, the other ones being similar. In this case $\mathcal{H}_{\mid \mathbb{P}_{y}} \cong\left(k(y)[s, t]^{\oplus 3}\right)^{\sim}$ and $\vartheta_{y}$ corresponds to the morphism $k(y)[s, t](-1)^{\oplus 3} \rightarrow$ $k(y)[s, t]^{\oplus 3}$ given, up to a suitable linear transformation, by the matrix

$$
\left(\begin{array}{ccc}
s & 0 & 0 \\
0 & t & 0 \\
0 & 0 & s+t
\end{array}\right) .
$$

Therefore it is easy to check that $\bar{\eta}_{y}: \mathcal{S}^{2} k(y)^{\oplus 3} \rightarrow \mathcal{S}^{2} k(y)^{\oplus 2}$ is given by the matrix

$$
\left(\begin{array}{llllll}
0 & 0 & 0 & 1 & 0 & 0 \\
1 & 0 & 0 & 1 & 0 & 1 \\
1 & 0 & 0 & 0 & 0 & 0
\end{array}\right),
$$

which has rank 3.

Suppose that $Y \cong \operatorname{spec}(A)$, where $A$ is local with maximal ideal $\mathfrak{M}$ ( $y$ the corresponding point of $Y), \mathcal{E} \cong A^{\oplus 3}$ with basis $\{u, v, w\}, \mathcal{F} \cong A^{\oplus 2}$, and let $\varrho: X \rightarrow$ $Y$ be a Gorenstein cover of degree 4. Theorem 2.4 implies that $X$ is given inside $\mathbb{P}(\mathcal{E}) \cong \mathbb{P}_{A}^{2}$ by two quadratic forms $a, b \in A[u, v, w]$. On the other hand, $X$ is affine; thus we can assume that $X_{y} \cap V_{+}(w)=\emptyset$. This means that $a$ and $b$ have no common factors modulo $(w)+\mathfrak{M}$; hence we can assume that $a=u^{2}+$ other terms in $u, v, w$ and $b=v^{2}+$ other terms in $u, v, w$. Finally, with proper linear transformations one easily gets that $X=\operatorname{spec}(R)$, where

$$
R:=\frac{A[u, v]}{\left(u^{2}+2 \alpha v+\beta, v^{2}+2 \gamma u+\delta\right)}
$$

for suitable $\alpha, \beta, \gamma, \delta \in A$.

If we set $L_{z}(y):=z y$ for $z \in R$ and $Q: R \times R \rightarrow A$ is the bilinear form defined by $Q(x, y):=\operatorname{Tr}(x y):=\operatorname{Tr}\left(L_{x y}\right)$, then an equation of $B_{\varrho}$ is $b=\operatorname{det}(Q)$ (see $[\mathrm{A}-\mathrm{K}]$, proposition 6.6). By (3.4), $\{1, u, v, u v\}$ is a basis of $R$ over $A$; then

$$
\begin{aligned}
b & =\left|\begin{array}{cccc}
4 & 0 & 0 & 12 \alpha \gamma \\
0 & -4 \beta & 12 \alpha \gamma & 8 \alpha \delta \\
0 & 12 \alpha \gamma & -4 \delta & 8 \beta \gamma \\
12 \alpha \gamma & 8 \alpha \delta & 8 \beta \gamma & 48 \alpha^{2} \gamma^{2}+4 \beta \delta
\end{array}\right| \\
& =-64\left(27 \alpha^{4} \gamma^{4}-18 \alpha^{2} \beta \gamma^{2} \delta-4 \beta^{3} \gamma^{2}-4 \alpha^{2} \delta^{3}-\beta^{2} \delta^{2}\right) .
\end{aligned}
$$


If $X:=\operatorname{spec}(R)$ where $R$ is defined by 3.4 , then $\vartheta$ is given by

$$
\left(\begin{array}{ccc}
s & 0 & \gamma t \\
0 & t & \alpha s \\
\gamma t & \alpha s & \beta s+\delta t
\end{array}\right) .
$$

It follows that $\bar{\eta}$ is induced by

$$
\left(\begin{array}{cccccc}
-\alpha^{2} & 0 & 0 & \beta & -\alpha & 0 \\
\beta & \alpha \gamma & 0 & \delta & 0 & 1 \\
\delta & 0 & -\gamma & -\gamma^{2} & 0 & 0
\end{array}\right),
$$

which has rank less than 3 at $y$ if and only if $\alpha \gamma, \alpha \delta, \beta \gamma, \beta \delta$ are all zero at $y$. More precisely, up to a permutation of $\alpha, \beta, \gamma$ and $\delta$ the only distinct cases are $\alpha, \beta, \gamma, \delta \in \mathfrak{M}$; then $S\left(X_{y}\right)=[1,1 ; ; 1], \alpha \notin \mathfrak{M}, \beta, \gamma, \delta \in \mathfrak{M}$, and then $S\left(X_{y}\right)=$ $[(2,1)]$, and $\alpha, \beta \notin \mathfrak{M}$ and $\gamma, \delta \in \mathfrak{M}$. Then $S\left(X_{y}\right)=[(1,1), 1]$.

With the above considerations in mind let us describe the singularities of $B_{\varrho}$, using (3.5) and Lemma 3.3 above.

Definition 3.6. Let $Y$ be smooth and integral and let $B \subseteq Y$ be a divisor through a point $y \in Y$.

Let $\mathfrak{M} \subseteq A:=\mathcal{O}_{Y, y}$ be the maximal ideal. We say that $y$ is a cuspidal double point (resp. a transverse double point) if the local equation of the tangent cone at $B$ around $y$ is $\ell^{2}$ (resp. $\ell_{1} \ell_{2}$ ) where $\ell \in \mathfrak{M} \backslash \mathfrak{M}^{2}$ (resp. $\ell_{1}, \ell_{2} \in \mathfrak{M} \backslash \mathfrak{M}^{2}$ are transversal).

A cuspidal double point is called ordinary if $(\ell, b) \subseteq \mathfrak{M}^{3} \backslash \mathfrak{M}^{4}$, non-ordinary otherwise.

Assume that both $X$ and $Y$ are smooth over a point $y \in Y$.

If $S\left(X_{y}\right)=[2,1], \varrho$ is either étale or analytically a double cover. Therefore $B_{\varrho}$ must be smooth.

If $S\left(X_{y}\right)=[3], \varrho$ is either étale or analytically a totally ramified triple cover. Therefore (see [Mi], section 4) $B_{\varrho}$ has at least a double point at $y$. If $y$ is exactly a double point then it is cuspidal (and, in general, ordinary).

If $S\left(X_{y}\right)=[(1,1), 1]$ then we can assume $\alpha, \beta \notin \mathfrak{M}$ and $\gamma, \delta \in \mathfrak{M}$ in (3.4). If $\gamma, \delta \in \mathfrak{M} \backslash \mathfrak{M}^{2}$ are not proportional the tangent cone at $y$ is $4 \beta^{3} \gamma^{2}+\beta^{2} \delta^{2}$, and so $y$ is a transverse double point. If either $\gamma$ and $\delta$ are proportional or at least one of them belongs to $\mathfrak{M}^{2}$, then it is easy to check by direct computation that $y$ is a non-ordinary cuspidal double point.

If $S\left(X_{y}\right)=[(2,1)]$, then we can assume $\alpha \notin \mathfrak{M}$ and $\beta, \gamma, \delta \in \mathfrak{M}$. In this case $y$ is at least a triple point.

Finally, if $S\left(X_{y}\right)=[1,1 ; ; 1]$, then $\alpha, \beta, \gamma, \delta \in \mathfrak{M}$. In this case $y$ is at least a fourfold point.

\section{The Discriminant of a COVer of Degree 4}

Let $\mathcal{E}, \mathcal{F}$ be locally free $\mathcal{O}_{Y}$-sheaves of ranks 3 and 2 respectively, and such that $\operatorname{det} \mathcal{E} \cong \operatorname{det} \mathcal{F}$. As in section 3 , we consider the isomorphism $\Psi$, and we identify $\vartheta:=\Psi(\eta)$ with a symmetric map $\check{\mathcal{G}}(-1) \stackrel{\vartheta}{\longrightarrow} \mathcal{G} \otimes \operatorname{det} \mathcal{G}^{-1}$. Taking the determinant of $\vartheta$ and applying the projection formula, we finally obtain a map of sets

$$
\Delta: H^{0}\left(Y, \check{\mathcal{F}} \otimes \mathcal{S}^{2} \mathcal{E}\right) \rightarrow H^{0}\left(Y, \mathcal{S}^{3} \mathcal{F} \otimes \operatorname{det} \mathcal{F}^{-1}\right)
$$

(recall that $\check{\mathcal{F}} \otimes \operatorname{det} \mathcal{F} \cong \mathcal{F})$. 
Let $\Phi_{3}$ be as in (2.2).

Definition 4.1. Let $\varrho: X \rightarrow Y$ be a Gorenstein cover of degree 4 corresponding to a section $\eta \in H^{0}\left(Y, \mathcal{S}^{2} \mathcal{E} \otimes \check{\mathcal{F}}\right)$. The scheme $\Delta(X):=D_{0}\left(\Phi_{3}(\Delta(\eta))\right) \subseteq \overline{\mathbb{P}}$ (resp. the map $\left.\Delta(\varrho):=\bar{\pi}_{\mid \Delta(X)}\right)$ is called the discriminant scheme of $X$ (resp. the discriminant map of $\varrho$ ).

If $X=\operatorname{spec}(R)$, where $R$ is defined by 3.4 , then

$$
\Delta(X) \cong \operatorname{proj}\left(\frac{A[s, t]}{\alpha^{2} t^{3}-\beta t^{2} s-\delta t s^{2}+\gamma^{2} s^{3}}\right) \subseteq \mathbb{P}_{A}^{1}
$$

Note that either $\Delta\left(X_{y}\right) \subseteq \mathbb{P}_{k(y)}^{1}$ is a subscheme of length 3 , or $\Delta\left(X_{y}\right) \cong \mathbb{P}_{k(y)}^{1}$. This second case is characterized by the vanishing of $\alpha, \beta, \gamma, \delta$ at the point $y$. In particular, $\Delta\left(X_{y}\right) \cong \mathbb{P}_{k(y)}^{1}$ if and only if

$$
X_{y} \cong \operatorname{spec}\left(\frac{k(y)[u, v]}{\left(u^{2}, v^{2}\right)}\right) .
$$

Remark 4.3. We identify $\Delta(\eta)$ with a map $\mathcal{S}^{3} \check{\mathcal{F}} \rightarrow \operatorname{det} \check{\mathcal{F}}$, and

$$
R_{\text {planar }}(\varrho)=\varrho^{-1}\left(D_{0}(\Delta(\eta))\right)
$$

by the above description. Thus $R_{\text {planar }}(\varrho)$ is closed, and either $\operatorname{codim}_{X}\left(R_{\text {planar }}(\varrho)\right)$ $\leq 4$ or $R_{\text {planar }}(\varrho)=\emptyset$, since $\operatorname{rk}\left(\mathcal{S}^{3} \mathcal{F} \otimes \operatorname{det} \mathcal{F}^{-1}\right)=4$.

Proposition 4.4. Let $\varrho: X \rightarrow Y$ be a Gorenstein cover of degree 4 with $Y$ integral and invariants $\mathcal{E}$ and $\mathcal{F}$. Then the following assertions are equivalent.

i) $\Delta(\varrho): \Delta(X) \rightarrow Y$ is a cover (of degree 3 ).

ii) $\Delta(\varrho): \Delta(X) \rightarrow Y$ is a Gorenstein cover.

iii) $R_{\text {planar }}(\varrho)=\emptyset$.

iv) For each $y \in Y$ the Zariski tangent space at $x \in X_{y}$ has dimension $\leq 1$.

Moreover, if $\Delta(\varrho)$ is a cover its Tschirnhausen module is $\check{\mathcal{F}}$, and if $Y$ is smooth at $y$ the branch loci of $\Delta(\varrho)$ and $\varrho, B_{\Delta(\varrho)}$ and $B_{\varrho}$, coincide as divisors around $y$.

Proof. i) and ii) are trivially equivalent since $\Delta(X) \subseteq \mathbb{P}(\mathcal{F})$ is a divisor. $\Delta(\varrho)$ is not a cover if and only if there is $y \in Y$ such that $\Delta\left(X_{y}\right)$ is not finite; hence i) and iii) are equivalent by the above description. The Zariski tangent space $T_{x}$ at $x \in X_{y}$ is the intersection of the Zariski tagent spaces of all the conics of $\mathbb{P}_{y}$ through $X_{y}$. Hence $T_{x}$ has dimension 2 if and only if all the conics through $X_{y}$ are singular at $x$, i.e. if and only if $\varrho$ is planar over $y$.

The equality $B_{\Delta(\varrho)}=B_{\varrho}$ must be checked locally; hence we assume $Y=\operatorname{spec}(A)$ and $X \cong \operatorname{spec}(R)$, where $R$ is defined by (3.4). Thus $B_{\varrho}=\operatorname{div}(b)$, where $b$ is defined by (3.5). On the other hand, if $\Delta(\varrho)$ is a cover we can also suppose that $\alpha$ does not vanish on $Y$. In this case $b$ coincides, up to the invertible factor $\alpha^{2}$, with the discriminant of the polynomial $p(t):=\alpha^{2} t^{3}-\beta t^{2}-\delta t+\gamma^{2}$ representing $\Delta(X)$ in $\operatorname{spec}(A[t])$.

From now on we will assume both $X$ and $Y$ are smooth. With these hypotheses we give some results about the reducibility, connectedness and smoothness of $\Delta(X)$.

First of all, note that there is a natural structure of regular conic bundle having $\Delta(X)$ as degeneration divisor (see [Sa], definitions 1.1 and 1.4 ), $p: B l_{X} \mathbb{P} \rightarrow \overline{\mathbb{P}}$, defined in the following way. If $x \in \mathbb{P} \backslash X$, let $y:=\pi(x)$ and let $C_{x}$ be the unique conic inside $\mathbb{P}_{y}$ containing both $x$ and $X_{y}$. Then $C_{x}$ corresponds to a point of $\overline{\mathbb{P}}_{y}$. 
Proposition 4.5. Let $\varrho: X \rightarrow Y$ be a cover of degree 4 with $X$ smooth and $Y$ smooth and integral. Then:

i) $\Delta(X)$ is reduced;

ii) if $\Delta(X)$ is reducible there is an invertible $\mathcal{O}_{Y}$-sheaf $\mathcal{M}$ and an epimorphism $\mathcal{F} \rightarrow \mathcal{M}$

iii) $\Delta(X)$ is singular at $x$ over $y \in Y$ if and only if $y \in R_{\text {even }}(\varrho) \cup R_{\text {planar }}(\varrho) . x$ corresponds to the conics of rank 1 cutting out $X_{y} \subseteq \mathbb{P}_{y} . x$ is a Gorenstein double point with tangent cone of rank at least 2 (briefly, a pseudo-node).

Proof. The statements i) and iii) follow from the above description, [Sa], proposition 1.8 , corollary 1.9, and the proof of proposition 1.2 iii) in [Bea].

Assume that $\Delta(X)=Z \cup Z^{\prime}$. Both $Z$ and $Z^{\prime}$ are Gorenstein (they are divisors inside $\overline{\mathbb{P}})$, and since the degree of $\Delta(\varrho)$ is 3 we can suppose that $\sigma:=\Delta(\varrho)_{\mid Z}: Z \rightarrow Y$ is generically finite of degree 1 ; hence $Z \subseteq \overline{\mathbb{P}}$ is a unisecant divisor. This implies the existence of an epimorphism $\mathcal{F} \rightarrow \mathcal{M}$ where $\mathcal{M}$ is invertible.

The following example shows that in Proposition 4.5 we cannot hope to prove the irreducibility of $\Delta(X)$.

Example 4.6. Let $Y$ be an integral smooth scheme, projective over $\mathbb{C}, \mathcal{L} \in \operatorname{Pic}(Y)$ globally generated, $\mathcal{F}:=\mathcal{L}^{2} \oplus \mathcal{L}^{2}, \mathcal{E}:=\mathcal{L} \oplus \mathcal{L} \oplus \mathcal{L}^{2}$. Let $u, v \in H^{0}\left(Y, \mathcal{E} \otimes \mathcal{L}^{-1}\right) \cong$ $H^{0}\left(\mathbb{P}, \mathcal{O}_{\mathbb{P}}(1) \otimes \pi^{*} \mathcal{L}^{-1}\right), w \in H^{0}\left(Y, \mathcal{E} \otimes \mathcal{L}^{-2}\right) \cong H^{0}\left(\mathbb{P}, \mathcal{O}_{\mathbb{P}}(1) \otimes \pi^{*} \mathcal{L}^{-2}\right)($ resp. $s, t \in$ $\left.H^{0}\left(Y, \mathcal{F} \otimes \mathcal{L}^{-2}\right) \cong H^{0}\left(\overline{\mathbb{P}}, \mathcal{O}_{\overline{\mathbb{P}}}(1) \otimes \bar{\pi}^{*} \mathcal{L}^{-2}\right)\right)$ be independent sections. For a general choice of $\beta, \delta \in H^{0}\left(Y, \mathcal{L}^{2}\right), \gamma \in H^{0}(Y, \mathcal{L})$ the subscheme $X \subseteq \mathbb{P}(\mathcal{E})$ defined by

$$
\eta:=\left(u^{2}+\beta w^{2}, v^{2}+2 \gamma u w+\delta w^{2}\right) \in H^{0}\left(Y, \check{\mathcal{F}} \otimes \mathcal{S}^{2} \mathcal{E}\right)
$$

is smooth and connected, since $h^{0}\left(Y, \mathcal{L}^{-1}\right)=0$. On the other hand $\Delta(X) \subseteq \mathbb{P}(\mathcal{F})$ has the equation

$$
p(s, t):=t\left(\beta s^{2}+\delta s t-\gamma^{2} t^{2}\right),
$$

and hence $\Delta(X)$ is reducible.

Corollary 4.7. Let $\varrho, X$ and $Y$ be as above. If $B_{\varrho}$ is reduced and $\Delta(X)$ is integral, then it is also normal. In particular, if $\operatorname{dim}(Y)=2$, then $\Delta(X)$ has at most isolated normal double points as singularities.

Proof. Since $\operatorname{Sing}(\Delta(X))$ lies exactly over $\operatorname{Sing}\left(B_{\varrho}\right)$ (see section 2) if $B_{\varrho}$ is reduced, then $\operatorname{Sing}(\Delta(X))$ has codimension at least 2. On the other hand, $\Delta(X)$ is CohenMacaulay. Hence the statement follows from Proposition 4.5 and [Sa], Proposition 1.

From now on we will assume that $\Delta(\varrho)$ is a cover, i.e., that $R_{\text {planar }}(\varrho)=\emptyset$.

Definition 4.8. Let $Y$ be an integral scheme and $Q \stackrel{q}{\rightarrow} Y$ a conic bundle. We define

$$
\operatorname{Sing}(q):=\{x \in Q \mid q \text { is not smooth at } x\}=\left\{x \in Q \mid x \in \operatorname{Sing}\left(q^{-1}(q(x))\right)\right\} .
$$

$\operatorname{Sing}(q)$ is closed in $Q$, and $q(\operatorname{Sing}(q))$ is exactly the discriminant curve of the conic bundle $Q$. Note that $\operatorname{Sing}(q) \neq \operatorname{Sing}(Q)$.

Now assume that $\Delta(X)$ is not connected. Then $\Delta(X)=Z \cup Z^{\prime}$ is a reduced scheme with $Z \cap Z^{\prime}=\emptyset$. We can suppose that $\sigma:=\Delta(\varrho)_{\mid Z}: Z \rightarrow Y$ is quasi-finite of degree 1 , so that it is a cover of degree 1 (see [Ha], exercises III 10.9 and III 
11.2), i.e., an isomorphism. In particular, $\sigma^{-1}$ is a section of $\bar{\pi}$. It follows that there exists a conic bundle $\mathbb{P} \supseteq Q \stackrel{q}{\rightarrow} Y$ containing $X$ such that $Q_{y}:=Q \cap \mathbb{P}_{y}$ is a conic of rank 2 for each $y \in Y$, and $\operatorname{Sing}(q) \cap X=\emptyset$ (recall that if $\mathfrak{b} \subseteq \mathbb{P}_{k}^{n}$ is a pencil of conics, then $C \in \mathfrak{b}$ corresponds to a simple root of the discriminant if and only if $\operatorname{Sing}(C) \cap B s(\mathfrak{b})=\emptyset)$. In particular, $q_{\mid \operatorname{Sing}(q)}$ : $\operatorname{Sing}(q) \rightarrow Y$ is an isomorphism (it is quasi-finite of degree 1); thus there is an invertible $\mathcal{O}_{Y}$-sheaf $\mathcal{L}$ such that the section $Y \stackrel{\sim}{\longrightarrow} \operatorname{Sing}(q) \subseteq \mathbb{P}$ corresponds to the exact sequence $0 \rightarrow \mathcal{E}_{0} \rightarrow \mathcal{E} \rightarrow \mathcal{L} \rightarrow 0$. Let $f: \mathbb{P} \backslash \operatorname{Sing}(q) \rightarrow \mathbb{P}\left(\mathcal{E}_{0}\right)$ be the induced dominant map (which is, fibrewise, the projection from the point $\operatorname{Sing}\left(Q_{y}\right)$ onto the line $\left.\mathbb{P}\left(\mathcal{E}_{0}\right)_{y}\right)$ and define $X_{0}:=f(X)$ (which is, fibrewise, $Q_{y} \cap \mathbb{P}\left(\mathcal{E}_{0}\right)_{y} \subseteq \mathbb{P}_{y}$ ). Let $\alpha:=f_{\mid X}: X \rightarrow X_{0}$. The projection $\mathbb{P}\left(\mathcal{E}_{0}\right) \rightarrow Y$ induces a double cover $\beta: X_{0} \rightarrow Y$ such that $\varrho=\beta \circ \alpha$. The ramification points of $\beta$ correspond to conics $Q_{y}$ of rank 1 ; hence $\beta$ is necessarily étale. Then we assume that

( $Q) \varrho$ does not factor as $\beta \circ \alpha$ with $\beta$ a non-trivial étale double cover of $Y$.

Condition $(\mathcal{Q})$ is obviously satisfied if $Y$ is simply connected.

If $(Q)$ is satisfied one gets that $X_{0}$ is trivial, and this allows us to find a decomposition $Q=\mathbb{P}_{1} \cup \mathbb{P}_{2}$ into two projective subbundles $\mathbb{P}_{i} \subseteq \mathbb{P}$ of rank 1. But, if this is the case, $X=X_{1} \cup X_{2}$, with $X_{i}:=X \cap \mathbb{P}_{i} \neq \emptyset$, since, generically, the fibre $X_{y}:=\varrho^{-1}(y)$ consists of four points in general position in $\pi^{-1}(y) \cong \mathbb{P}_{k}^{2}$.

Proposition 4.9. Let $\varrho: X \rightarrow Y$ be a cover of degree 4 with $X$ and $Y$ smooth and integral and $R_{\text {planar }}(\varrho)=\emptyset$. If $Y$ satisfies $(Q)$ then $\Delta(X)$ is connected.

Corollary 4.10. If $\varrho, X$ and $Y$ are as above, then $h^{0}(Y, \check{\mathcal{F}})=0$.

Proof. The number of connected components of $\Delta(X)$ is

$$
h^{0}\left(\Delta(X), \mathcal{O}_{\Delta(X)}\right)=h^{0}\left(Y, \mathcal{O}_{Y}\right)+h^{0}(Y, \check{\mathcal{F}}) .
$$

Corollary 4.11. If $\varrho, X$ and $Y$ are as above and $B_{\varrho}$ is reduced (resp. has at most ordinary cuspidal double points as singularities), then $\Delta(X)$ is integral (resp. smooth).

Proof. We already know that $\Delta(X)$ is reduced. If $\Delta(X) \subseteq \mathbb{P}(\mathcal{F})$ were reducible then we would have $\operatorname{dim}(\operatorname{Sing}(\Delta(X))) \geq \operatorname{dim}(X)-1$ (since $\Delta(X)$ is connected). On the other hand,

$$
\begin{aligned}
\operatorname{Sing}(\Delta(X)) & \subseteq \Delta(\varrho)^{-1}\left\{y \in Y \mid S\left(X_{y}\right)=[(1,1), 1],[(2,1)]\right\} \\
& \subseteq \Delta(\varrho)^{-1}\left(\operatorname{Sing}\left(B_{\Delta(\varrho)}\right)\right)=\Delta(\varrho)^{-1}\left(\operatorname{Sing}\left(B_{\varrho}\right)\right) .
\end{aligned}
$$

Hence $\operatorname{dim}(\operatorname{Sing}(\Delta(X))) \leq \operatorname{dim}\left(\operatorname{Sing}\left(B_{\varrho}\right)\right) \leq \operatorname{dim}(X)-2$.

If $B_{\varrho}$ has at most ordinary cuspidal double points as singularities, then the smoothness of $\Delta(X)$ follows from section 3 .

Both Proposition 4.9 and its Corollaries 4.10 and 4.11 are sharp, as the following easy examples show.

Example 4.12. Consider Example 4.6. As we saw, $\Delta(X)$ is in any case reducible. On the other hand, using the expression of $b$ given by (3.5), one finds that the branch locus $B_{\varrho}$ has global equation

$$
b=4 \beta^{3} \gamma^{2}+\beta^{2} \delta^{2}=\left(4 \beta \gamma^{2}+\delta^{2}\right) \beta^{2},
$$

which is not reduced. 
Example 4.13. Consider an elliptic curve $Y$ over $\mathbb{C}$, and let $\beta: X_{0} \rightarrow Y$ be a double étale cover with $X_{0}$ smooth and connected. Then there is an invertible sheaf $\mathcal{L}$ such that $\mathcal{L}^{2} \cong \mathcal{O}_{Y}$ and $\beta_{*} \mathcal{O}_{X_{0}} \cong \mathcal{O}_{Y} \oplus \mathcal{L}^{-1}$. Now let $\mathcal{M}$ be an invertible and globally generated $\mathcal{O}_{Y}$-sheaf and let $\alpha: X \rightarrow X_{0}$ be any smooth and connected double cover with $\alpha_{*} \mathcal{O}_{X} \cong \mathcal{O}_{X_{0}} \oplus \beta^{*} \mathcal{M}^{-1}$. The morphism $\varrho:=\beta \circ \alpha: X \rightarrow Y$ is a Gorenstein cover of degree 4 whose Tschirnahausen module is dual to $\mathcal{E}:=\mathcal{L} \oplus \mathcal{M} \oplus$ $\mathcal{L} \otimes \mathcal{M}$. Moreover, $\omega_{X \mid k} \cong \omega_{X \mid X_{0}} \otimes \omega_{X_{0} \mid Y} \cong \varrho^{*}(\mathcal{L} \otimes \mathcal{M})$. It is not difficult to check that $\mathcal{F} \cong \mathcal{L}^{2} \oplus \mathcal{M}^{2} \cong \mathcal{O}_{Y} \oplus \mathcal{M}^{2}$. Hence $h^{0}(Y, \check{\mathcal{F}})=h^{0}\left(Y, \mathcal{O}_{Y}\right)+h^{0}\left(Y, \mathcal{M}^{-2}\right)=1$, so that $\Delta(X)$ is not connected. Note that, since $X$ is smooth, $\Delta(\varrho): \Delta(X) \rightarrow Y$ is actually a cover of degree 3 .

\section{Singularities of the Discriminant}

In section 4 we gave a first rough description of $\operatorname{Sing}(\Delta(X))$. In this section we want to study the nature of the singularities of the discriminant of a cover $\varrho: X \rightarrow Y$ of degree 4 under the hypothesis of genericity. We will assume that $X$ is smooth and $Y$ is smooth and integral.

To that end we inspect the construction of the discriminant as the degeneration divisor of the conic bundle $p: B l_{X} \mathbb{P} \rightarrow \overline{\mathbb{P}}$ used in the previous section. For each $x \in \mathbb{P} \backslash X$ let $y:=\pi(x)$, and let $C_{x}$ be the unique conic contained in $\mathbb{P}_{y}$ and containing both $x$ and $X_{y}$. Then $C_{x} \in \overline{\mathbb{P}}_{y}$. We have a commutative diagram

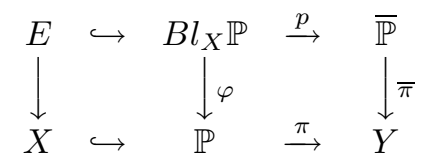

where $\varphi$ is the natural projection. Since the fibres of $p$ are conics, it follows that $p$ is a Gorenstein morphism (of relative dimension 1). Hence, so is $\bar{\pi} \circ p$, and the relative dualizing sheaf $\omega_{B l_{X} \mathbb{P} \mid Y}$ is invertible. Moreover, by semicontinuity, $\mathcal{H}^{\prime}:=p_{*} \omega_{B l_{X} \mathbb{P} \mid Y}^{-1}$ is a locally free $\mathcal{O}_{\overline{\mathbb{P}}}$ sheaf of rank 2 and the natural epimorphism $p^{*} \mathcal{H}^{\prime} \rightarrow \omega_{B l_{X} \mathbb{P} \mid Y}$ induces an embedding $B l_{X} \mathbb{P} \hookrightarrow \widetilde{\mathbb{P}}:=\mathbb{P}\left(\mathcal{H}^{\prime}\right)$ factoring $p$ through the natural projection $\widetilde{p}: \widetilde{\mathbb{P}} \rightarrow \overline{\mathbb{P}}$. In particular, $p: B l_{X} \mathbb{P} \rightarrow \overline{\mathbb{P}}$ is an embedded conic in the sense of [Sa], 1.5.

Since $p$ is induced by the relative conics through $X$, it follows that $p^{*} \mathcal{O}_{\overline{\mathbb{P}}}(1) \cong$ $\varphi^{*} \mathcal{O}_{\mathbb{P}}(2) \otimes \mathcal{O}_{B l_{X} \mathbb{P}}(-E)$. In particular,

$$
\omega_{B l_{X} \mathbb{P} \mid Y} \cong \varphi^{*} \omega_{\mathbb{P} \mid Y} \otimes \mathcal{O}_{B l_{X} \mathbb{P}}(E) \cong p^{*} \mathcal{O}_{\overline{\mathbb{P}}}(-1) \otimes \varphi^{*} \mathcal{O}_{\mathbb{P}}(-1) \otimes \varphi^{*} \pi^{*} \operatorname{det} \mathcal{E} .
$$

Then $\mathcal{H}^{\prime} \cong \mathcal{O}_{\overline{\mathbb{P}}}(1) \otimes p_{*} \varphi^{*}\left(\mathcal{O}_{\mathbb{P}}(1) \otimes \pi^{*} \operatorname{det} \mathcal{E}^{-1}\right)$. Since $\pi, \bar{\pi}$ and $p$ are flat, then $p_{*} \varphi^{*} \cong \bar{\pi}^{*} \pi_{*}$ as functors (see [Ha], proposition III 9.3); hence $\mathcal{H}^{\prime} \cong \mathcal{H}:=\mathcal{O}_{\overline{\mathbb{P}}}(1) \otimes$ $\bar{\pi}^{*}\left(\mathcal{E} \otimes \operatorname{det} \mathcal{E}^{-1}\right) . B l_{X} \mathbb{P}$ has an $\mathcal{O}_{\widetilde{\mathbb{P}}}-$ resolution of the form

$$
0 \rightarrow \mathcal{O}_{\widetilde{\mathbb{P}}}(-2) \otimes \widetilde{p}^{*} \mathcal{M}^{-1} \rightarrow \mathcal{O}_{\widetilde{\mathbb{P}}} \rightarrow \mathcal{O}_{B l_{X} \mathbb{P}} \rightarrow 0
$$

where $\mathcal{M} \cong \mathcal{O}_{\overline{\mathbb{P}}}(-1) \otimes \bar{\pi}^{*} \operatorname{det} \mathcal{F}$ (see [Sa], 1.5 and 1.6). Note that $\mathcal{S}^{2} \mathcal{H} \otimes \mathcal{M} \cong$ $\bar{\pi}^{*}\left(\mathcal{S}^{2} \mathcal{E} \otimes \operatorname{det} \mathcal{E}^{-1}\right) \otimes \mathcal{O}_{\overline{\mathbb{P}}}(1) ;$ in particular, $\bar{\pi}_{*}\left(\mathcal{S}^{2} \mathcal{H} \otimes \mathcal{M}\right) \cong \check{\mathcal{F}} \otimes \mathcal{S}^{2} \mathcal{E}$, and there is a natural isomorphism

$$
\Phi: H^{0}\left(Y, \check{\mathcal{F}} \otimes \mathcal{S}^{2} \mathcal{E}\right) \stackrel{\sim}{\longrightarrow} H^{0}\left(\overline{\mathbb{P}}, \mathcal{S}^{2} \mathcal{H} \otimes \mathcal{M}\right) \cong \operatorname{Hom}_{\mathcal{O}_{\overline{\mathbb{P}}}}^{\text {sym }}(\check{\mathcal{H}}, \mathcal{H} \otimes \mathcal{M})
$$


The symmetric map $\vartheta=\Phi(\eta): \check{\mathcal{H}} \rightarrow \mathcal{H} \otimes \mathcal{M}$ yields an exact sequence

$$
0 \rightarrow \check{\mathcal{H}} \stackrel{\vartheta}{\rightarrow} \mathcal{H} \otimes \mathcal{M} \rightarrow \mathcal{P} \rightarrow 0
$$

where $\mathcal{P}$ is supported on $\Delta(X)$. Notice that $\Delta(X)=D_{2}(\vartheta)$ (by construction) and $\operatorname{Sing}(\Delta(X))=D_{1}(\vartheta)$ (Proposition 4.5 iii)).

With this in mind we can use the results of [Ba] to describe the singularities of $\Delta(X)$.

Proposition 5.3. Let $\mathcal{E}, \mathcal{F}$ be locally free sheaves of ranks 3 and 2 respectively over an integral and smooth scheme $Y$ of dimension 2.

If $\mathcal{S}^{2} \mathcal{E} \otimes \check{\mathcal{F}}$ is globally generated, then the discriminant $\Delta(X)$ of each general cover $\varrho: X \rightarrow Y$ of degree 4 with invariants $\mathcal{E}$ and $\mathcal{F}$ is smooth in codimension 1 and has at most nodes as singularities.

Proof. The natural map $\bar{\pi}^{*} \mathcal{F} \rightarrow \mathcal{O}_{\overline{\mathbb{P}}}(1)$ induces an epimorphism $\bar{\pi}^{*}\left(\mathcal{S}^{2} \mathcal{E} \otimes \check{\mathcal{F}}\right) \rightarrow$ $\mathcal{S}^{2} \mathcal{H} \otimes \mathcal{M}$. If $\mathcal{S}^{2} \mathcal{E} \otimes \check{\mathcal{F}}$ is globally generated, then the same is true for $\mathcal{S}^{2} \mathcal{H} \otimes \mathcal{M}$. Thus the proposition follows from proposition 1 of [Ba].

Proposition 5.4. Let $\varrho: X \rightarrow Y$ be a cover of degree 4 with $X$ smooth, $Y$ smooth, integral and projective, and invariants $\mathcal{E}, \mathcal{F}$. If $\Delta(X)$ is smooth in codimension 1 , then $\operatorname{Sing}(\Delta(X))$ represents the class of

$$
4 \bar{\pi}^{*}\left(c_{2}(\mathcal{E})-c_{2}(\mathcal{F})\right) \cdot \xi-4 \bar{\pi}^{*} c_{3}(\mathcal{E}),
$$

$\xi \in \operatorname{Pic}(\overline{\mathbb{P}})$ being the tautological class. In particular, if $\operatorname{dim}(Y)=2$ and $\Delta(X)$ has at most nodes as singularities, then

$$
\text { \# } \operatorname{Sing}(\Delta(X))=4\left(c_{2}(\mathcal{E})-c_{2}(\mathcal{F})\right) .
$$

Proof. To prove the statement we consider formula 9 of [Ba]. In this case $r=3$, $c_{i}=c_{i}(\mathcal{H}), \lambda=c_{1}(\mathcal{M})$, where $\mathcal{M} \cong \mathcal{O}_{\bar{\pi}}(-1) \otimes \bar{\pi}^{*} \operatorname{det} \mathcal{E}$ and $\mathcal{H}=\bar{\pi}^{*} \mathcal{E} \otimes \mathcal{M}^{-1}$. A direct substitution in Barth's formula then proves that $\operatorname{Sing}(\Delta(X))$ represents

$$
4\left(\xi^{3}-\bar{\pi}^{*} c_{1}(\mathcal{E}) \cdot \xi^{2}+\bar{\pi}^{*} c_{2}(\mathcal{E}) \cdot \xi-\bar{\pi}^{*} c_{3}(\mathcal{E})\right) .
$$

Since $\xi^{3}-\bar{\pi}^{*} c_{1}(\mathcal{F}) \cdot \xi^{2}+\bar{\pi}^{*} c_{2}(\mathcal{F}) \cdot \xi=0$ and $c_{1}(\mathcal{E})=c_{1}(\mathcal{F})$, we get formula (5.4.1). Formula (5.4.2) follows by projecting via $\bar{\pi}_{*}$ and the fact that the singularities of $\Delta(X)$ are nodes.

Example 5.5. Let $X$ be an Enriques surface not containing nodal curves. There always exists a cover $\varrho: X \rightarrow \mathbb{P}_{k}^{2}$ of degree 4 whose invariants are $\mathcal{E} \cong \mathcal{O}_{\mathbb{P}_{k}^{2}}(2)^{\oplus 3}$ and the kernel $\mathcal{F}$ of an epimorphism $\mathcal{O}_{\mathbb{P}_{k}^{2}}(4)^{\oplus 3} \rightarrow \mathcal{O}_{\mathbb{P}_{k}^{2}}(6)$ (see section 6 of [C-E] and [Ve]). In particular, $c_{2}(\mathcal{E})=12=c_{2}(\mathcal{F})$. Since in this case $\mathcal{S}^{2} \mathcal{E} \otimes \check{\mathcal{F}}$ is globally generated, for each such general $\varrho$ the discriminant $\Delta$ is smooth.

Indeed, A. Verra proved in [Ve] that the branch locus of $\varrho$ is a reduced curve of degree 12 having ordinary cuspidal points as singularities; thus $R_{\text {even }}(\varrho)=\emptyset$ by Corollary 4.11.

\section{The trigonal CONSTRUCtion}

In this section we want to investigate the connection between the discriminant of a cover of degree 4 and a generalization of the well known trigonal construction due to S. Recillas (see $[\mathrm{Re}]$ ).

We maintain the notations of sections 4 and 5 . The first step is to study the sheaf $\mathcal{P}$ fitting into the sequence (5.2). 
Definition 6.1. Let $\Delta$ be a scheme defined over a field of characteristic $p \neq 2, \mathcal{L}$ an invertible $\mathcal{O}_{\Delta}$-sheaf and $\mathcal{P}$ a coherent reflexive $\mathcal{O}_{\Delta}$-sheaf. We say that $\mathcal{P}$ is an $\mathcal{L}$-quadratic sheaf if there exists a symmetric isomorphism

$$
\sigma: \mathcal{P} \stackrel{\sim}{\longrightarrow} \check{\mathcal{P}} \otimes \mathcal{L} \cong \mathcal{H o m}_{\mathcal{O}_{\Delta}}(\mathcal{P}, \mathcal{L}) .
$$

Lemma 6.2. If $\Delta(X)$ is normal, then the $\mathcal{O}_{\Delta(X)}$-sheaf $\mathcal{P}$ fitting in the sequence 5.2 above is an $\omega_{\Delta(X) \mid Y^{2}}^{2}$ quadratic sheaf.

$\mathcal{P}$ is not invertible exactly on $\operatorname{Sing}(\Delta(X))$.

Proof. Taking the tensor product of sequence 5.2 with itself, we obtain

$$
(\check{\mathcal{H}} \otimes \mathcal{H} \otimes \mathcal{M}) \oplus(\mathcal{H} \otimes \mathcal{M} \otimes \check{\mathcal{H}}) \stackrel{\delta_{1}}{\longrightarrow}(\mathcal{H} \otimes \mathcal{M}) \otimes(\mathcal{H} \otimes \mathcal{M}) \rightarrow \mathcal{P} \otimes \mathcal{P} \rightarrow 0,
$$

where $\delta_{1}(\alpha \otimes a, b \otimes \beta)=\vartheta(\alpha) \otimes a+b \otimes \vartheta(\beta)$ and which is exact (use the spectral sequence of a double complex). On the other hand, let $\mathfrak{S}_{2}:=\{1, i\}$. The map $T_{0}:=1+i$ acts on $(\mathcal{H} \otimes \mathcal{M}) \otimes(\mathcal{H} \otimes \mathcal{M})$ sending $a \otimes b$ to $a \otimes b+b \otimes a$. In particular, $T_{0}$ extends to an endomorphism $T$ of (6.2.1) whose image is a direct summand, which is isomorphic to

$$
\check{\mathcal{H}} \otimes \mathcal{H} \otimes \mathcal{M} \stackrel{d_{1}}{\longrightarrow} \mathcal{S}^{2}(\mathcal{H} \otimes \mathcal{M}) \rightarrow \mathcal{S}^{2} \mathcal{P} \rightarrow 0
$$

via the canonical projection. It follows that 6.2 .2 is exact.

The adjoint of $\vartheta$ maps $\mathcal{H} \otimes \mathcal{M}$ to $\check{\mathcal{H}}(\Delta(X))$; hence it induces a morphism $\phi_{1}$ : $\mathcal{S}^{2}(\mathcal{H} \otimes \mathcal{M}) \rightarrow \mathcal{M}(\Delta(X))$. Denoting by $\phi_{2}: \check{\mathcal{H}} \otimes \mathcal{H} \otimes \mathcal{M}$ the usual contraction, we then get a commutative diagram with exact rows

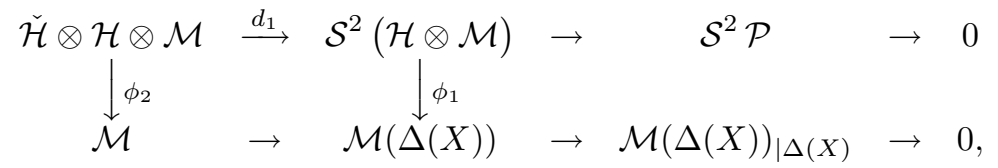

giving rise to a map $\phi_{0}: \mathcal{S}^{2} \mathcal{P} \rightarrow \mathcal{M}(\Delta(X))_{\mid \Delta(X)} \cong \omega_{\Delta(X) \mid Y}^{2}$, hence to a morphism $\sigma: \mathcal{P} \rightarrow \check{\mathcal{P}} \otimes \omega_{\Delta(X) \mid Y}^{2}$. Since $\operatorname{Sing}(\Delta(X))=D_{1}(\vartheta)$, we see that $\phi_{1}$ is surjective outside $\operatorname{Sing}(\Delta(X))$ and the same is true for $\phi_{0}$. It follows that $\sigma$ is an isomorphism outside $\operatorname{Sing}(\Delta(X))$, since $\mathcal{P}$ is invertible there (see [Ba], Lemma 5 ).

Taking the dual of sequence (5.2) twisted by $\mathcal{M}$, one gets

$$
0 \rightarrow \check{\mathcal{H}} \rightarrow \mathcal{H} \otimes \mathcal{M} \rightarrow \mathcal{E} x t_{\mathcal{O}_{\overline{\mathbb{P}}}}^{1}(\mathcal{P}, \mathcal{M}) \rightarrow 0 .
$$

The short exact sequence $0 \rightarrow \mathcal{M} \rightarrow \mathcal{M}(\Delta(X)) \rightarrow \mathcal{M}(\Delta(X))_{\mid \Delta(X)} \rightarrow 0$ yields an isomorphism $\partial: \mathcal{E} x t_{\mathcal{O}_{\overline{\mathbb{P}}}}^{1}(\mathcal{P}, \mathcal{M}) \stackrel{\sim}{\longrightarrow} \check{\mathcal{P}} \otimes \omega_{\Delta(X) \mid Y}^{2}$. In particular, locally on $Y, \sigma$ lifts to a chain map between sequences (5.2) and (6.2.3), and any two such maps are homotopic. For degree reasons any homotopy must be zero, so we obtain a commutative diagram

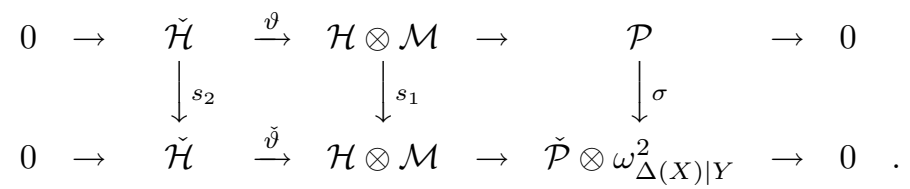

Sequences (5.2) and (6.2.3) are minimal, so $s_{1}$ and $s_{2}$ are isomorphisms outside $\operatorname{Sing}(\Delta(X))$. The normality of $\Delta(X)$ then implies that they are both isomorphisms everywhere, so the same must be true for $\sigma$. 
$\mathcal{P}$ is reflexive. Indeed, $\mathcal{P}$ is torsion-free, so the natural map $\mu: \mathcal{P} \rightarrow \mathcal{P}^{\sim}$ is injective. On the other hand, $\mathcal{P}$ and $\mathcal{P}^{\sim}$ are isomorphic via $\left(\sigma^{t}\right)^{-1} \circ \sigma$. Thus $\mathcal{P}$ and $\mathcal{P}^{\sim}$ have the same Hilbert function, and it follows that $\operatorname{coker}(\mu)=0$.

Since $\mathcal{P}$ is reflexive and $\sigma$ is induced by $\phi_{0}$ it follows that $\sigma$ is symmetric, i.e. $\mathcal{P}$ is an $\omega_{\Delta(X) \mid Y}^{2}$-quadratic sheaf.

Finally, $\mathcal{P}$ is invertible outside $\operatorname{Sing}(\Delta(X))$. On the other hand, if $x \in$ $\operatorname{Sing}(\Delta(X))$ then $y:=\pi(x)$ is even or planar (Proposition 4.5 iii)). In the first case $X_{y}=C \cap D$, where $C=V_{+}\left(u^{2}+v^{2}\right)$ and $D=V_{+}\left(w^{2}\right)$; thus $t^{2} s$ is an equation for $\Delta(X)_{y} \subseteq \overline{\mathbb{P}}_{y}$ and $\vartheta_{y}$ is defined by the matrix

$$
\left(\begin{array}{lll}
t & 0 & 0 \\
0 & t & 0 \\
0 & 0 & s
\end{array}\right)
$$

Note that $D \in \Delta(X)_{y}$ corresponds to $s=1$. We conclude that

$$
\operatorname{dim}_{k(D)}(\mathcal{P} \otimes k(D))=2,
$$

and so $\mathcal{P}$ cannot be invertible at $D$. The cases $S\left(X_{y}\right)=[(2,1)],[1,1 ; ; 1]$ can be treated analogously.

Definition 6.3. Let $Y$ be integral, defined over a field of characteristic $p \neq 2$, and let $\mathcal{E}$ and $\mathcal{F}$ be locally free $\mathcal{O}_{Y}$-sheaves of rank 3 and 2 respectively.

Let $\mathcal{T}_{Y, \mathcal{E}, \mathcal{F}}^{4}$ denote the set of Gorenstein covers $X \stackrel{\varrho}{\rightarrow} Y$ of degree 4 with invariants $\mathcal{E}, \mathcal{F}$ and such that $X$ is smooth and $\operatorname{codim}_{X}\left(R_{\text {even }}(\varrho)\right) \geq 2, R_{\text {planar }}(\varrho)=\emptyset$.

Let $\mathcal{R}_{Y, \mathcal{E}, \mathcal{F}}^{3}$ denote the set of pairs $(\mathcal{P}, \Delta \stackrel{\tau}{\rightarrow} Y)$ such that $\tau$ is a Gorenstein cover of degree 3 with invariant $\mathcal{F}, \Delta$ is normal with at most pseudo-nodes as singularities, and $\mathcal{P}$ is an $\omega_{\Delta \mid Y}^{2}$-quadratic sheaf which is invertible exactly outside $\operatorname{Sing}(\Delta)$ and such that $\tau_{*} \mathcal{P} \cong \mathcal{E}$.

Up to now we have shown how to associate to each $X \stackrel{\varrho}{\rightarrow} Y \in \mathcal{T}_{Y, \mathcal{E}, \mathcal{F}}^{4}$ an element $\operatorname{Trig}(X \stackrel{\varrho}{\rightarrow} Y) \in \mathcal{R}_{Y, \mathcal{E}, \mathcal{F}}^{3}$.

Definition 6.4. The map

$$
\text { Trig: } \mathcal{T}_{Y, \mathcal{E}, \mathcal{F}}^{4} \rightarrow \mathcal{R}_{Y, \mathcal{E}, \mathcal{F}}^{3}
$$

defined above is called the "trigonal construction".

Theorem 6.5. The map Trig is bijective.

Proof. We only need to show how to recover a Gorenstein cover of degree 4 from a fixed pair $(\mathcal{P}, \Delta \stackrel{\tau}{\rightarrow} Y)$.

First of all, assume that $Y=\operatorname{spec}(k)$ is a point, i.e. $\overline{\mathbb{P}} \cong \mathbb{P}_{k}^{1}$. Then $H^{0}(Y, \mathcal{P}) \cong$ $k^{\oplus 3}, \mathcal{P}$ is 0 -regular since $\operatorname{dim}(\Delta)=0$ and it is supported on $\Delta$. In particular the natural maps $H^{0}(Y, \mathcal{P}) \otimes H^{0}\left(Y, \mathcal{O}_{\mathbb{P}_{k}^{1}}(n)\right) \rightarrow H^{0}(Y, \mathcal{P}(n))$ are surjective for each $n \geq 0$. Thus there exists an epimorphism $\varphi: \mathcal{O}_{\mathbb{P}_{k}^{1}}^{\oplus 3} \rightarrow \mathcal{P}$ whose kernel is free, say $\operatorname{ker}(\varphi) \cong \bigoplus_{i=1}^{3} \mathcal{O}_{\mathbb{P}_{k}^{1}}\left(-\alpha_{i}\right)$, as follows from the Auslander-Buchsbaum formula and the decomposition theorem of Grothendieck. It follows that an exact sequence of the form

$$
0 \rightarrow \bigoplus_{i=1}^{3} \mathcal{O}_{\mathbb{P}_{k}^{1}}\left(-\alpha_{i}\right) \rightarrow \mathcal{O}_{\mathbb{P}_{k}^{1}}^{\oplus 3} \stackrel{\varphi}{\longrightarrow} \mathcal{P} \rightarrow 0
$$


is defined. Since the map $H^{0}(\varphi)$ is an isomorphism, taking the cohomologies of (6.5.1) one obtains $\alpha_{i}=1$ for $i=1,2,3$.

Now consider an arbitrary scheme $Y$ and let $\mathcal{M}$ and $\mathcal{H}$ be as in section 5 . One obtains the exact sequence

$$
0 \rightarrow \mathcal{K} \stackrel{\theta}{\rightarrow} \mathcal{H} \otimes \mathcal{M} \rightarrow \mathcal{P} \rightarrow 0,
$$

whose restriction to $\overline{\mathbb{P}}_{y}$ coincides with (6.5.1). Since $h^{1}\left(\overline{\mathbb{P}}_{y}, \mathcal{K}_{\mid \overline{\mathbb{P}}_{y}}\right)=0$ and $h^{0}\left(\overline{\mathbb{P}}_{y}, \mathcal{K}_{\mid \overline{\mathbb{P}}_{y}}\right)=3$, thus $R^{1} \bar{\pi}_{*} \mathcal{K}(1)=0$ and $\mathcal{N}:=\bar{\pi}_{*} \mathcal{K}(1)$ is locally free of rank 3. Moreover, the natural map $\bar{\pi}^{*} \mathcal{N} \rightarrow \mathcal{K}(1)$ is an isomorphism since this is true fibrewise.

The symmetric map $\sigma$ induces $\mathcal{S}^{2} \mathcal{P} \rightarrow \omega_{\Delta \mid Y}^{2} \cong \mathcal{M}(\Delta)_{\mid \Delta}$, hence a diagram

$$
\begin{aligned}
& 0 \rightarrow \Lambda^{2} \mathcal{K} \stackrel{d_{2}}{\longrightarrow} \mathcal{K} \otimes \mathcal{H} \otimes \mathcal{M} \stackrel{d_{1}}{\longrightarrow} \mathcal{S}^{2}(\mathcal{H} \otimes \mathcal{M}) \rightarrow \underset{\left.\right|_{\phi}}{\rightarrow} \quad \begin{array}{lll}
\mathcal{S}^{2} \mathcal{P} & \rightarrow & 0
\end{array} \\
& 0 \quad \rightarrow \quad \mathcal{M} \quad \rightarrow \quad \mathcal{M}(\Delta) \quad \rightarrow \quad \mathcal{M}(\Delta)_{\mid \Delta} \rightarrow 0 .
\end{aligned}
$$

Locally on the base the map $\phi_{0}$ can be lifted to a chain map, and any two such maps are homotopic. On the other hand, since $\mathcal{K} \cong \bar{\pi}^{*} \mathcal{N}(-1)$, for degree reasons each homotopy must be zero, so we obtain a well defined chain map and a commutative diagram

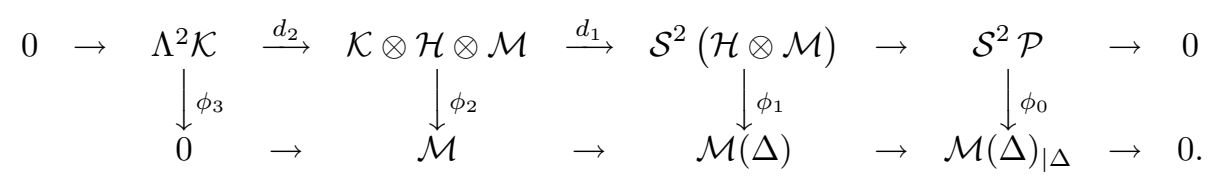

The map $\phi_{2}$ induces a morphism $s_{1}: \mathcal{H} \rightarrow \check{\mathcal{K}}$, defined locally by $\left\langle s_{1}(\alpha), \beta\right\rangle:=$ $\phi_{2}(\alpha \otimes \beta)$. Let $s_{2}$ be the transpose of $s_{1}$ : we claim that $s_{1} \circ \theta=\check{\theta} \circ s_{2}$. Indeed, we have locally

$$
0=\phi_{3}(\alpha \wedge \beta)=\phi_{2} \circ d_{2}(\alpha \wedge \beta)=\phi_{2}(\alpha \otimes \theta(\beta)-\beta \otimes \theta(\alpha)) .
$$

Thus

$$
\left\langle s_{1} \circ \theta(\alpha), \beta\right\rangle=\phi_{2}(\beta \otimes \theta(\alpha))=\phi_{2}(\alpha \otimes \theta(\beta))=\left\langle s_{2}(\alpha), \theta(\beta)\right\rangle=\left\langle\check{\theta} \circ s_{2}(\alpha), \beta\right\rangle .
$$

It follows that there exists a chain map

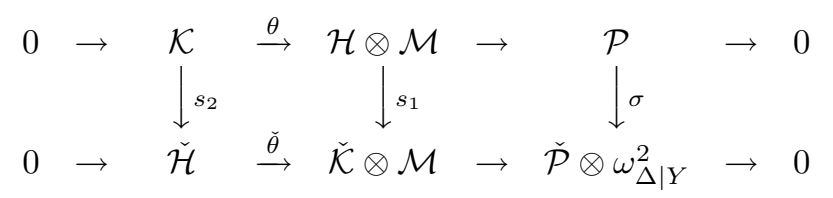

extending the isomorphism $\sigma$. By construction each row of the above diagram is minimal; thus the maps $s_{1}$ and $s_{2}$ are isomorphisms and we get the exact sequence

$$
0 \rightarrow \check{\mathcal{H}} \stackrel{\vartheta}{\rightarrow} \mathcal{H} \otimes \mathcal{M} \rightarrow \mathcal{P} \rightarrow 0,
$$

where $\vartheta:=\theta \circ s_{2}^{-1}$. Notice that $\vartheta=\check{\vartheta}$. Hence we obtain $\eta \in H^{0}\left(Y, \check{\mathcal{F}} \otimes \mathcal{S}^{2} \mathcal{E}\right)$ via the isomorphism $\Phi^{-1}$ defined in section 5 .

We claim that $\eta$ has the right codimension at each point $y \in Y$. Indeed, $\eta$ defines a subscheme $X:=D_{0}(\eta) \subseteq \mathbb{P}$ which is fibrewise over $y \in Y$ the base locus of a pencil of conics $\mathfrak{b}_{y}$ whose discriminant is $\Delta_{y}$. Since $\tau$ is a cover we have $\Delta_{y} \neq \mathbb{P}_{k(y)}^{1}$. 
Hence $\mathfrak{b}_{y}$ has no fixed components, and $\varrho:=\pi_{\mid X}: X \rightarrow Y$ is a Gorenstein cover of degree 4 without planar points.

It remains to prove the smoothness of $X$. Since $\mathcal{P}$ is not invertible exactly on $\operatorname{Sing}(\Delta)$, it follows that $\varrho(\operatorname{Sing}(X)) \subseteq \varrho\left(R_{\text {even }}(\varrho)\right)=\tau(\operatorname{Sing}(\Delta))$. Let $y \in$ $\varrho\left(R_{\text {even }}(\varrho)\right)$ be such that $S\left(X_{y}\right)$ is either $[(2,1)]$ or $[(1,1), 1]$. Then $X_{y}=C \cap D$ where $C=V_{+}\left(u^{2}\right)$ and $D=V_{+}\left(v^{2}+2 u\right)\left(\operatorname{so} \operatorname{Supp}\left(X_{y}\right)=(0,0)\right)$ in the first case or $C=V_{+}\left(u^{2}\right)$ and $D=V_{+}\left(v^{2}-1\right)\left(\right.$ so $\left.\operatorname{Supp}\left(X_{y}\right)=\{(0, \pm 1)\}\right)$ in the second. Locally on $Y$ the subscheme $X \subseteq \mathbb{P}$ is given by $\operatorname{spec}(R)$, where $R$ is as in (3.4), and either $\alpha(y)=\beta(y)=\delta(y)=0$ and $\gamma(y)=1$ in the first case, or $\alpha(y)=\beta(y)=\gamma(y)=0$ and $\delta(y)=-1$ in the second (see (2.2) and the description above). Then $\Delta$ is given by (4.2) locally on $Y$. Moreover, $C \in \operatorname{Sing}(\Delta)$ corresponds to $s=0$. Let $\left\{y_{1}, \ldots, y_{n}\right\}$ be a system of local regular parameters around $y$, and assume that $X$ is singular over $y$. If $S\left(X_{y}\right)=[(2,1)]$, one can easily check that the jacobian matrix of $X$ at $u=v=0$ is

$$
\left(\begin{array}{lll}
0 & 0 & \frac{\partial \beta}{\partial y_{i}} \\
2 & 0 & \frac{\partial \delta}{\partial y_{i}}
\end{array}\right)_{i=1, \ldots, n},
$$

whence $\left(\frac{\partial \beta}{\partial y_{i}}\right)_{\mid y}=0$. If $S\left(X_{y}\right)=[(1,1), 1]$, the jacobian matrix at $u=0, v= \pm 1$ is

$$
\left(\begin{array}{ccc}
0 & 0 & \pm 2 \frac{\partial \alpha}{\partial y_{i}}+\frac{\partial \beta}{\partial y_{i}} \\
0 & \pm 2 & \frac{\partial \delta}{\partial y_{i}}
\end{array}\right)_{i=1, \ldots, n},
$$

whence $\left(\frac{\partial \alpha}{\partial y_{i}}\right)_{\mid y}= \pm 2 i\left(\frac{\partial \beta}{\partial y_{i}}\right)_{\mid y}=0$. In both the cases the tangent cone at $C \in \Delta$ would have rank at most 1 .

Remark 6.6. It is not difficoult to verify that giving a $\omega_{\Delta \mid Y}^{2}$-quadratic sheaf $\mathcal{P}$ on $\Delta$ is equivalent to giving a finite morphism $\Delta^{\prime} \stackrel{\tau^{\prime}}{\longrightarrow} \Delta$ of degree 2 branched exactly at $\operatorname{Sing}(\Delta)$. Indeed, we define the $\mathcal{O}_{\Delta}$-algebra $\mathcal{A}:=\mathcal{O}_{\Delta} \oplus\left(\mathcal{P} \otimes \omega_{\Delta \mid Y}^{-1}\right)$ with the multiplication induced by $\sigma$ and consider the canonical projection $\Delta^{\prime}:=\operatorname{Spec}(\mathcal{A})$ onto $\Delta$.

We give the following classical example.

Example 6.7. Let $X:=\mathbb{P}_{k}^{1} \times_{k} \mathbb{P}_{k}^{1}$. The linear system $\mathcal{O}_{X}(1,2)$ induces an embedding $i: X \hookrightarrow \mathbb{P}_{k}^{5}$. Projecting $i(X)$ onto a non-intersecting plane $\alpha$, we finally get a cover $\varrho: X \rightarrow \mathbb{P}_{k}^{2}$ of degree 4 . The invariants of $\varrho$ are $\mathcal{E} \cong \mathcal{O}_{\mathbb{P}_{k}^{2}}(1)^{\oplus 3}$ and $\mathcal{F} \cong \mathcal{O}_{\mathbb{P}_{k}^{2}}(1) \oplus \mathcal{O}_{\mathbb{P}_{k}^{2}}(2)$. It follows from Proposition 5.3 and formula (5.4.2) that for a general projection the discriminant $\Delta$ has exactly 4 nodes as singularities. If $\overline{\mathbb{P}} \cong B l_{r} \mathbb{P}_{k}^{3} \stackrel{\varphi}{\longrightarrow} \mathbb{P}_{k}^{3}$ is the blow-up of $\mathbb{P}_{k}^{3}$ along a line $r$, then $\varphi(\Delta) \subseteq \mathbb{P}_{k}^{3}$ is a cubic and $\varphi_{\mid \Delta}$ is an isomorphism. We conclude that $\varphi(\Delta)$ is a Cayley cubic. On the other hand, we also have a double cover $\Delta^{\prime} \stackrel{\tau^{\prime}}{\longrightarrow} \Delta$ branched along $\operatorname{Sing}(\Delta)$. One has $\tau_{*} \tau^{\prime}{ }_{*} \mathcal{O}_{\Delta^{\prime}} \cong \mathcal{O}_{\mathbb{P}_{k}^{2}} \oplus \mathcal{O}_{\mathbb{P}_{k}^{2}}(-1)^{\oplus 4} \oplus \mathcal{O}_{\mathbb{P}_{k}^{2}}(-2)$. $\Delta^{\prime}$ is a Del Pezzo sextic in $\mathbb{P}_{k}^{6}$, and $\tau \tau^{\prime}$ is the projection from a space $\beta \subseteq \mathbb{P}_{k}^{6}$ of dimension 3 .

It is a classical result that all the Cayley cubic surfaces arise in this way.

\section{Covers of $\mathbb{P}_{\mathbb{C}}^{n}$.}

We defined $R_{\text {planar }}(\varrho)$ in section 4 for any cover $\varrho: X \rightarrow Y$ of degree 4 with both $X$ and $Y$ smooth and integral. In this section we want to restrict ourselves to the 
case $Y \cong \mathbb{P}_{\mathbb{C}}^{n}$, and we will deal with the behaviour of $\varrho$ with respect to $R_{\text {planar }}(\varrho)$ when $n \geq 5$. In this case there always exist points $y \in Y$ of total ramification (see $[\mathrm{G}-\mathrm{L}])$.

Proposition 7.1. Let $X$ be integral and smooth, $n \geq 5$, and let $\varrho: X \rightarrow \mathbb{P}_{\mathbb{C}}^{n}$ be a cover of degree 4 . Then

i) there is $k \geq 2$ such that $\omega_{X \mid \mathbb{P}_{\mathbb{C}}^{n}} \cong \varrho^{*} \mathcal{O}_{\mathbb{P}_{\mathbb{C}}^{n}}(k)$;

ii) for each line $r \subseteq \mathbb{P}_{\mathbb{C}}^{n}$ there are $k_{2} \leq k_{1}<k$, depending on $r$, such that $k_{1}+k_{2}=k, \mathcal{E}_{\mid r} \cong \mathcal{O}_{r}(k) \oplus \mathcal{O}_{r}\left(k_{1}\right) \oplus \mathcal{O}_{r}\left(k_{2}\right)$ and $\mathcal{F}_{\mid r} \cong \mathcal{O}_{r}\left(2 k_{1}\right) \oplus \mathcal{O}_{r}\left(2 k_{2}\right)$;

iii) $c_{1}(\mathcal{F})=c_{1}(\mathcal{E})=2 k, c_{2}(\mathcal{F})=4 c_{2}(\mathcal{E})-k^{2}$.

Proof. If $n \geq 5$ then $\omega_{X \mid \mathbb{P}_{\mathbb{C}}^{n}} \cong \varrho^{*} \mathcal{O}_{\mathbb{P}_{\mathbb{C}}^{n}}(k)$ for some $k \neq 0$ (see [La], proposition 3.1). Moreover, the isomorphisms $\mathcal{O}_{\mathbb{P}_{\mathbb{C}}^{n}}(-k) \oplus \mathcal{E}(-k) \cong\left(\varrho_{*} \omega_{X \mid \mathbb{P}_{\mathrm{C}}^{n}}\right)(-k) \cong \varrho_{*} \mathcal{O}_{X} \cong \mathcal{O}_{\mathbb{P}_{\mathbb{C}}^{n}} \oplus \check{\mathcal{E}}$ give rise to a factorization of the identity on $\mathcal{O}_{\mathbb{P}_{\mathbb{C}}^{n}}$ as $\mathcal{O}_{\mathbb{P}_{\mathbb{C}}^{n}} \stackrel{i}{\rightarrow} \mathcal{O}_{\mathbb{P}_{\mathbb{C}}^{n}}(-k) \oplus \mathcal{E}(-k) \stackrel{p}{\rightarrow}$ $\mathcal{O}_{\mathbb{P}_{\mathbb{C}}^{n}}$. Since $h^{0}\left(\mathbb{P}_{\mathbb{C}}^{n}, \mathcal{O}_{\mathbb{P}_{\mathbb{C}}^{n}}(-k)\right)+h^{0}\left(\mathbb{P}_{\mathbb{C}}^{n}, \mathcal{E}(-k)\right)=h^{0}\left(X, \mathcal{O}_{X}\right)=1$, it follows that $k \geq 1, h^{0}\left(\mathbb{P}_{\mathbb{C}}^{n}, \mathcal{O}_{\mathbb{P}_{\mathbb{C}}^{n}}(-k)\right)=0$ and $h^{0}\left(\mathbb{P}_{\mathbb{C}}^{n}, \mathcal{E}(-k)\right)=1$. Thus one can split both $i$ and $p$ through $\mathcal{E}(-k)$; hence $\mathcal{E} \cong \mathcal{O}_{\mathbb{P}^{n}}^{n}(k) \oplus \mathcal{E}_{0}$, where rk $\mathcal{E}_{0}=2$ and $c_{1}(\mathcal{E})=c_{1}\left(\mathcal{E}_{0}\right)+k$. One has an embedding $\mathbb{P}_{0}:=\mathbb{P}\left(\mathcal{E}_{0}\right) \subseteq \mathbb{P}$, and $\mathcal{O}_{\mathbb{P}}\left(\mathbb{P}_{0}\right) \cong \mathcal{O}_{\mathbb{P}}(1) \otimes \pi^{*} \mathcal{O}_{\mathbb{P}_{c}^{n}}(-k)$. Since $X_{0}:=\mathbb{P}_{0} \cap X$ is effective and $\mathcal{O}_{X}\left(X_{0}\right) \cong \mathcal{O}_{\mathbb{P} \mid X} \cong \mathcal{O}_{X}$, we see that $X_{0}=\emptyset$, and sequence (2.1) becomes

$$
0 \rightarrow \pi_{0}^{*} \operatorname{det} \mathcal{E}(-4) \rightarrow \pi_{0}^{*} \mathcal{F}(-2) \rightarrow \mathcal{O}_{\mathbb{P}_{0}} \rightarrow 0,
$$

where $\pi_{0}:=\pi_{\mid \mathbb{P}_{0}}$. Twisting (7.1.1) by $\mathcal{O}_{\mathbb{P}_{0}}(2)$ and taking its direct image via $\pi_{0}$, we get

$$
0 \rightarrow \mathcal{F} \rightarrow \mathcal{S}^{2} \mathcal{E}_{0} \rightarrow \mathcal{O}_{\mathbb{P}_{\mathbb{C}}^{n}}(k) \rightarrow 0 .
$$

In particular, $3 c_{1}\left(\mathcal{E}_{0}\right)=c_{1}\left(\mathcal{S}^{2} \mathcal{E}_{0}\right)=c_{1}(\mathcal{F})+k=c_{1}(\mathcal{E})+k=c_{1}\left(\mathcal{E}_{0}\right)+2 k$, i.e. $c_{1}\left(\mathcal{E}_{0}\right)=k$ and $c_{1}(\mathcal{E})=c_{1}(\mathcal{F})=2 k$.

For each line $r \subseteq \mathbb{P}_{\mathbb{C}}^{n}$ the scheme $\varrho^{-1}(r)$ is connected (theorem 7.1 of [Jo]). Since we wish to deal with $\mathcal{E}_{\mid r}$ and $\mathcal{F}_{\mid r}$ we can assume that $n=1$. One has $\mathcal{E}_{0} \cong \mathcal{O}_{\mathbb{P}_{\mathbb{C}}^{1}}\left(k_{1}\right) \oplus \mathcal{O}_{\mathbb{P}_{\mathbb{C}}^{1}}\left(k_{2}\right), k_{1} \geq k_{2} \geq 1$ ( $X$ is connected $)$ and $k_{1}+k_{2}=k$; then $k>k_{1} \geq 1$ (this completes the proof of i)). Let $\mathcal{F} \cong \mathcal{O}_{\mathbb{P}_{\mathbb{C}}^{1}}\left(h_{1}\right) \oplus \mathcal{O}_{\mathbb{P}_{\mathbb{C}}^{1}}\left(h_{2}\right), h_{1} \geq h_{2}$. Sequence (7.1.2) becomes

$$
0 \rightarrow \mathcal{O}_{\mathbb{P}_{\mathbb{C}}^{1}}\left(h_{1}\right) \oplus \mathcal{O}_{\mathbb{P}_{\mathbb{C}}^{1}}\left(h_{2}\right) \rightarrow \mathcal{O}_{\mathbb{P}_{\mathbb{C}}^{1}}\left(2 k_{1}\right) \oplus \mathcal{O}_{\mathbb{P}_{\mathbb{C}}^{1}}\left(2 k_{2}\right) \oplus \mathcal{O}_{\mathbb{P}_{\mathbb{C}}^{1}}(k) \stackrel{a}{\rightarrow} \mathcal{O}_{\mathbb{P}_{\mathbb{C}}^{1}}(k) \rightarrow 0 .
$$

The matrix of $a$ is

$$
\left(a_{2 k_{1}-k}, a_{2 k_{2}-k}, a_{0}\right) \text {, }
$$

where $a_{i} \in H^{0}\left(\mathbb{P}_{\mathbb{C}}^{1}, \mathcal{O}_{\mathbb{P}_{\mathbb{C}}^{1}}(i)\right)$. If $a_{0} \neq 0$, then (7.1.3) splits, and ii) is proved.

If $a_{0}=0$ there is a monomorphism $\mathcal{O}_{\mathbb{P}_{\mathbb{C}}^{1}}(k) \longmapsto \mathcal{O}_{\mathbb{P}_{\mathbb{C}}^{1}}\left(h_{1}\right) \oplus \mathcal{O}_{\mathbb{P}_{\mathbb{C}}^{1}}\left(h_{2}\right)$. In particular $h_{1} \geq k=k_{1}+k_{2}$; hence $h_{1} \geq 2 k_{2}$. If $h_{1}=2 k_{2}$ then $h_{2}=2 k-h_{1}=2 k-2 k_{2}=2 k_{1}$.

Assume $h_{1}>2 k_{2}$, and let $w \in H^{0}\left(\mathbb{P}_{\mathbb{C}}^{1}, \mathcal{E}(-k)\right) \cong H^{0}\left(\mathbb{P}, \mathcal{O}_{\mathbb{P}}(1) \otimes \pi^{*} \mathcal{O}_{\mathbb{P}_{\mathbb{C}}^{1}}(-k)\right)$, $v \in H^{0}\left(\mathbb{P}_{\mathbb{C}}^{1}, \mathcal{E}\left(-k_{1}\right)\right) \cong H^{0}\left(\mathbb{P}, \mathcal{O}_{\mathbb{P}}(1) \otimes \pi^{*} \mathcal{O}_{\mathbb{P}_{\mathbb{C}}^{1}}\left(-k_{1}\right)\right)$ and $u \in H^{0}\left(\mathbb{P}_{\mathbb{C}}^{1}, \mathcal{E}\left(-k_{2}\right)\right) \cong$ $H^{0}\left(\mathbb{P}, \mathcal{O}_{\mathbb{P}}(1) \otimes \pi^{*} \mathcal{O}_{\mathbb{P}_{\mathbb{C}}^{1}}\left(-k_{2}\right)\right)$ be independent sections. Then $\mathbb{P}_{0}=V_{+}(w) \subseteq \mathbb{P}$, and $X_{0}:=\mathbb{P}_{0} \cap X \subseteq \mathbb{P}$ is defined by a system of equations of the form

$$
\begin{aligned}
w & =\alpha_{2 k_{2}-h_{1}} u^{2}+\alpha_{k_{1}+k_{2}-h_{1}} u v+\alpha_{2 k_{1}-h_{1}} v^{2} \\
& =\alpha_{2 k_{2}-h_{2}} u^{2}+\alpha_{k_{1}+k_{2}-h_{2}} u v+\alpha_{2 k_{1}-h_{2}} v^{2}=0
\end{aligned}
$$


where $\alpha_{i} \in H^{0}\left(\mathbb{P}_{\mathbb{C}}^{1}, \mathcal{O}_{\mathbb{P}_{\mathbb{C}}^{1}}(i)\right)$. Since $h_{1}>2 k_{2}$, then $\alpha_{2 k_{2}-h_{1}}=0$. If $2 k_{2} \neq h_{2}$, there is $y \in \mathbb{P}_{\mathbb{C}}^{1}$ such that $\alpha_{2 k_{2}-h_{2}}(y)=0$ and $(y,[1,0,0]) \in X_{0} \neq \emptyset$, which is absurd. We conclude that $h_{1}=2 k_{1}, h_{2}=2 k_{2}$.

iii) is an easy computation from sequence (7.1.2).

Corollary 7.2. Let $\varrho, X, n$ be as above. If either $\mathcal{F}$ or $\mathcal{E}_{0}$ is uniform, then $\mathcal{E} \cong \mathcal{O}_{\mathbb{P}_{\mathbb{C}}^{n}}\left(k_{1}\right) \oplus \mathcal{O}_{\mathbb{P}_{\mathbb{C}}^{n}}\left(k_{2}\right) \oplus \mathcal{O}_{\mathbb{P}_{\mathbb{C}}^{n}}\left(k_{1}+k_{2}\right), \mathcal{F} \cong \mathcal{O}_{\mathbb{P}_{\mathbb{C}}^{n}}\left(2 k_{1}\right) \oplus \mathcal{O}_{\mathbb{P}_{\mathbb{C}}^{n}}\left(2 k_{2}\right)$ and $\omega_{X \mid \mathbb{P}_{\mathbb{C}}^{n}} \cong$ $\varrho^{*} \mathcal{O}_{\mathbb{P}^{n}}\left(k_{1}+k_{2}\right)$.

Moreover, the natural map $\varrho_{i}^{*}: H^{i}\left(\mathbb{P}_{\mathbb{C}}^{n}, \mathbb{C}\right) \rightarrow H^{i}(X, \mathbb{C})$ is an isomorphism for $0 \leq i \leq n-1$ and a monomorphism (but not an isomorphism) for $i=n$.

Proof. If $n \geq 5$ and either $\mathcal{F}$ or $\mathcal{E}_{0}$ is uniform, then the other one is also uniform and hence they both split (see [O-S-S], theorem I 3.2.3).

Now fix $k_{1}$ and $k_{2}$. Covers $\varrho: X \rightarrow \mathbb{P}_{\mathbb{C}}^{n}$ with $X$ smooth and invariants $\mathcal{E}$ and $\mathcal{F}$ correspond to sections $\eta$ of a suitable open set $\mathcal{U} \subseteq H^{0}\left(\mathbb{P}_{\mathbb{C}}^{n}, \check{\mathcal{F}} \otimes \mathcal{S}^{2} \mathcal{E}\right.$ ) (see Theorems 2.4 and 2.5). We claim that $\mathcal{U} \neq \emptyset$. Indeed, let $\alpha: Y \rightarrow \mathbb{P}_{\mathbb{C}}^{n}$ and $\beta: X^{\prime} \rightarrow$ $Y$ be two covers of degree 2 defined by general sections in $H^{0}\left(\mathbb{P}_{\mathbb{C}}^{n}, \mathcal{O}_{\mathbb{P}_{\mathbb{C}}^{n}}\left(k_{2}\right)\right)$ and $H^{0}\left(Y, \alpha^{*} \mathcal{O}_{\mathbb{P}_{\mathbb{C}}^{n}}\left(k_{1}\right)\right)$ respectively, so that one can assume both $Y$ and $X^{\prime}$ smooth. It is easy to check that $\varrho^{\prime}:=\alpha \circ \beta: X^{\prime} \rightarrow \mathbb{P}_{\mathbb{C}}^{n}$ corresponds to a section $\eta^{\prime} \in \mathcal{U}$. Obviously $\mathcal{U}$ is connected, and there is a smooth family

$$
\mathcal{X}:=\bigcup_{\eta \in \mathcal{U}}\left(D_{0}\left(\Phi_{4}(\eta)\right), \eta\right) \subseteq \mathbb{P} \times_{\mathbb{C}} \mathcal{U} \stackrel{\chi}{\rightarrow} \mathcal{U}
$$

whose special fibres are $X$ and $X^{\prime}$. In particular $b_{i}\left(\chi^{-1}(\eta)\right)$ is constant on $\mathcal{U}$; hence it suffices to prove that $b_{i}\left(\mathbb{P}_{\mathbb{C}}^{n}\right)=b_{i}\left(X^{\prime}\right), 0 \leq i \leq n-1$, and $b_{n}\left(\mathbb{P}_{\mathbb{C}}^{n}\right)<b_{n}\left(X^{\prime}\right)$, since in any case $\varrho_{i}^{*}$ are monomorphisms because $\varrho$ is finite. Since both $\alpha$ and $\beta$ are cyclic covers with ample branch loci, one has isomorphisms $H^{i}\left(\mathbb{P}_{\mathbb{C}}^{n}, \mathbb{C}\right) \stackrel{\alpha_{i}^{*}}{\longrightarrow} H^{i}(Y, \mathbb{C}) \stackrel{\beta_{i}^{*}}{\longrightarrow}$ $H^{i}\left(X^{\prime}, \mathbb{C}\right)$ for $0 \leq i \leq n-1$ (see [La], Theorem 2.1). Finally, $b_{n}\left(\mathbb{P}_{\mathbb{C}}^{n}\right)=b_{n}\left(X^{\prime}\right)$ if and only if both $\alpha_{i}^{*}$ and $\beta_{i}^{*}$ are isomorphisms. Then, from theorem 2.7 of [Wi], we get that $Y$ would be a hyperquadric in $\mathbb{P}_{\mathbb{C}}^{n+1}$ with $n$ odd. But now it follows from theorem 2.9 of [Wi] that $\beta_{n}^{*}$ can never be an isomorphism.

Remark 7.3. Let $\varrho: X \rightarrow \mathbb{P}_{\mathbb{C}}^{n}$ be a cover of degree $d \leq n-1$ with $X$ integral and

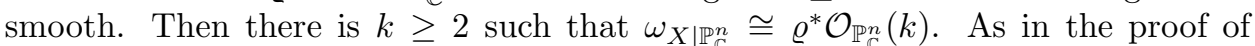
Proposition 7.1 one obtains that $\mathcal{E} \cong \mathcal{E}_{0} \oplus \mathcal{O}_{\mathbb{P}_{c}^{n}}(k)$, where $\mathcal{E}_{0}$ is ample and rk $\mathcal{E}_{0}=$ $d-2$. Since $X \cap \mathbb{P}\left(\mathcal{E}_{0}\right)=\emptyset$, the embedding $X \hookrightarrow \mathbb{P}$ gives rise to $X \hookrightarrow \mathbb{V}:=\mathbb{V}\left(\mathcal{E}_{0}\right)$; hence the natural maps $\varrho_{i}^{*}: H^{i}\left(\mathbb{P}_{\mathbb{C}}^{n}, \mathbb{C}\right) \rightarrow H^{i}(X, \mathbb{C})$ are isomorphisms if $i \leq n+2-d$. This slightly improves Theorems 1 and 2.1 of [La].

Remark 7.4. Of course, if Hartshorne's conjecture holds, the uniformity condition above is superfluous.

We now apply the results proved above in order to get a description of covers of $\mathbb{P}_{\mathbb{C}}^{n}$ with $R_{\text {planar }}(\varrho)=\emptyset$. In particular we will obtain the "local-global" criterion 1.3. We will make use of the theorems proved in $[\mathrm{Fj}]$.

Lemma 7.5. Let $\varrho: X \rightarrow Y$ be a Gorenstein cover of degree 4 with $R_{\text {planar }}(\varrho)=$ $\emptyset$. Assume that $\varrho$ is not étale, $Y$ is integral, smooth and projective over $\mathbb{C}$ with $\operatorname{dim}(Y) \geq 4, \Delta(X)$ is irreducible, every non-zero effective divisor on $Y$ is ample, and for each cycle $\zeta$ of codimension 2 on $Y$ one has $\zeta^{2} \cdot c_{1}(\mathcal{E})^{n-4} \geq 0$. Then $\mathcal{F} \cong \mathcal{L} \oplus \mathcal{L}^{2}$ for some ample invertible $\mathcal{O}_{Y}$-sheaf $\mathcal{L}$. 
Proof. Proposition 4.4 and Remark 4.3 imply that $\Delta(\varrho): \Delta(X) \rightarrow Y$ is a Gorenstein cover of degree 3 and the map $\Delta(\eta): \mathcal{S}^{3} \breve{\mathcal{F}} \rightarrow \operatorname{det} \check{\mathcal{F}}$ is surjective. It follows that we can imitate word for word the proof of [Fj], theorem 2.2 and corollary 2.3, taking into account that $c_{1}(\mathcal{E})=c_{1}(\mathcal{F})$. In particular, either $\Delta(\varrho)$ is étale (and the same is true for $\varrho$ by Proposition 4.4), or $\mathcal{F} \cong \mathcal{L} \oplus \mathcal{L}^{2}$ for some ample $\mathcal{L} \in \operatorname{Pic}(Y)$ (see $[\mathrm{Fj}]$, proof of theorem 2.1; see also [La]).

We now prove a more precise form of Theorem 1.3 which was stated in the introduction.

Theorem 7.6. Let $\varrho: X \rightarrow \mathbb{P}_{\mathbb{C}}^{n}$ be a cover of degree 4 , with $X$ smooth and $n \geq 5$.

If $R_{\text {planar }}(\varrho) \neq \emptyset$, then $\operatorname{codim}_{X}\left(R_{\text {planar }}(\varrho)\right) \leq 4$ and the branch locus $B_{\varrho}$ of $\varrho$ has at least a fourfold point.

$R_{\text {planar }}(\varrho)=\emptyset$ if and only if there is $h>0$ such that $X$ is a quadrisecant of the line bundle associated to $\mathcal{O}_{\mathbb{P}_{\mathbb{C}}^{n}}(h)$. In particular, $\mathcal{F} \cong \mathcal{O}_{\mathbb{P}_{\mathbb{C}}^{n}}(2 h) \oplus \mathcal{O}_{\mathbb{P}_{\mathbb{C}}^{n}}(4 h)$, $\mathcal{E} \cong \mathcal{O}_{\mathbb{P}_{\mathbb{C}}^{n}}(h) \oplus \mathcal{O}_{\mathbb{P}_{\mathbb{C}}^{n}}(2 h) \oplus \mathcal{O}_{\mathbb{P}_{\mathbb{C}}^{n}}(3 h)$, and $\omega_{X \mid \mathbb{P}_{\mathbb{C}}^{n}} \cong \varrho^{*} \mathcal{O}_{\mathbb{P}_{\mathbb{C}}^{n}}(3 h)$.

Proof. The first assertion follows from Remark 4.3 and section 3.

Obviously, if $X$ is a quadrisecant of a line bundle, then $R_{\text {planar }}(\varrho)=\emptyset$. Conversely, let $R_{\text {planar }}(\varrho)=\emptyset$. Then the structure of the sheaves $\mathcal{E}, \mathcal{F}$ and $\omega_{X \mid \mathbb{P}_{\mathrm{c}}^{n}}$ follows from Corollary 7.2 if we prove that $\mathcal{F} \cong \mathcal{L} \oplus \mathcal{L}^{2}$ for some $\mathcal{L} \in \operatorname{Pic}\left(\mathbb{P}_{\mathbb{C}}^{n}\right)$. Assume that $\Delta(X)$ is irreduciblel then we can apply Lemma 7.5.

It remains only to study the case when $\Delta(X)$ is reducible. In this case by Proposition 4.5 ii) there is an exact sequence $0 \rightarrow \mathcal{O}_{\mathbb{P}_{\mathbb{C}}^{n}}(a) \rightarrow \mathcal{F} \rightarrow \mathcal{O}_{\mathbb{P}_{\mathbb{C}}^{n}}(b) \rightarrow 0$. Since $H^{1}\left(\mathbb{P}_{\mathbb{C}}^{n}, \mathcal{O}_{\mathbb{P}_{\mathbb{C}}^{n}}(a-b)\right)=0$, then $\mathcal{F} \cong \mathcal{O}_{\mathbb{P}_{\mathbb{C}}^{n}}(a) \oplus \mathcal{O}_{\mathbb{P}_{\mathbb{C}}^{n}}(b)$ with, for example, $a \leq b$. Thus, $\Delta(X)$ is represented in $\overline{\mathbb{P}}$ by the single equation $\alpha_{2 a-b} s^{3}+\alpha_{a} s^{2} t+\alpha_{b} s t^{2}+\alpha_{2 b-a} t^{3}=$ 0 , where $s \in H^{0}\left(\mathbb{P}_{\mathbb{C}}^{n}, \mathcal{F}(-a)\right) \cong H^{0}\left(\overline{\mathbb{P}}, \mathcal{O}_{\overline{\mathbb{P}}} \otimes \bar{\pi}^{*} \mathcal{O}_{\mathbb{P}_{\mathbb{C}}^{n}}(-a)\right), t \in H^{0}\left(\mathbb{P}_{\mathbb{C}}^{n}, \mathcal{F}(-b)\right) \cong$ $H^{0}\left(\overline{\mathbb{P}}, \mathcal{O}_{\overline{\mathbb{P}}} \otimes \bar{\pi}^{*} \mathcal{O}_{\mathbb{P}_{\mathbb{C}}^{n}}(-b)\right)$ are independent sections and $\alpha_{i} \in H^{0}\left(\mathbb{P}_{\mathbb{C}}^{n}, \mathcal{O}_{\mathbb{P}_{\mathbb{C}}^{n}}(i)\right)$. Since $n \geq 5$, if $b \neq 2 a$ there is $y \in \mathbb{P}_{\mathbb{C}}^{n}$ such that $\alpha_{i}(y)=0$, which contradicts the assumption $R_{\text {planar }}(\varrho)=\emptyset$.

Let $u \in H^{0}\left(\mathbb{P}_{\mathbb{C}}^{n}, \mathcal{E}(-h)\right), v \in H^{0}\left(\mathbb{P}_{\mathbb{C}}^{n}, \mathcal{E}(-2 h)\right)$ be independent sections. Up to a suitable linear transformation on the variables $u$ and $v$, the above discussion implies that $X \subseteq \mathbb{P}$ has equations

$$
\begin{aligned}
& \alpha(u, v):=\alpha_{0} u^{2}+\alpha_{3 h} v+\alpha_{4 h}, \\
& \beta(u, v):=\beta_{0}^{\prime} u+\beta_{0}^{\prime \prime} v^{2}+\beta_{h} v+\beta_{2 h},
\end{aligned}
$$

where $\alpha_{i}, \beta_{i} \in H^{0}\left(\mathbb{P}_{\mathbb{C}}^{n}, \mathcal{O}_{\mathbb{P}_{\mathbb{C}}^{n}}(i)\right)$. Obviously $\beta_{0}^{\prime \prime} \neq 0$. If $\beta_{0}^{\prime}=0$ then

$$
\emptyset \neq\left\{y \in \mathbb{P}_{\mathbb{C}}^{n} \mid \alpha_{3 h}(y)=\alpha_{4 h}(y)=\beta_{h}(y)=\beta_{2 h}(y)=0\right\} \subseteq \varrho\left(R_{\text {planar }}(\varrho)\right) ;
$$

hence we can assume $\beta_{0}^{\prime} \neq 0$. Using $\beta$, we can write $u$ explicitly as a quadratic polynomial in the variable $v$. Substituting in $\alpha$, we get $X$ as a quadrisecant of the line bundle associated to $\mathcal{O}_{\mathbb{P}_{\mathbb{C}}^{n}}(h)$.

Remark 7.7. In $[\mathrm{G}-\mathrm{L}]$ the loci $R_{\ell}(\varrho):=\left\{x \in X \mid\right.$ the local degree at $x$ is $e_{\varrho}(x) \geq$ $\ell+1\}$ are defined. In particular, $R_{\text {planar }}(\varrho) \subseteq R_{3}(\varrho)$. In theorem 1 of $[\mathrm{G}-\mathrm{L}]$ it is proved that $\operatorname{codim}_{X}\left(R_{\ell}(\varrho)\right) \leq \ell$. In our setting, $R_{\ell}(\varrho) \neq \emptyset$ if $\ell \leq 3$.

Example 7.8. The bound on $n$ is sharp. Let us consider a globally generated invertible sheaf $\mathcal{L}$ on $\mathbb{P}_{\mathbb{C}}^{n}$ with $n \leq 4$, and define $\mathcal{F} \cong \mathcal{L} \oplus \mathcal{L}^{2}, \mathcal{E} \cong \mathcal{L}^{\oplus 3}$. Since $\mathcal{H}:=\check{\mathcal{F}} \otimes \mathcal{S}^{2} \mathcal{E}$ is globally generated, for any general $\eta \in H^{0}(Y, \mathcal{H})$ one has a smooth 
subscheme $X:=D_{0}\left(\Phi_{4}(\eta)\right) \subseteq \mathbb{P}$. Moreover, any such $X$ has global equations of the form

$$
\alpha u v+\beta u w+\gamma v^{2}+\delta v w+\epsilon w^{2}=u^{2}-v w=0 ;
$$

hence any such $\eta$ has the right codimension at each point $y \in \mathbb{P}_{\mathbb{C}}^{n}$, i.e. $\varrho:=\pi_{\mid X}: X \rightarrow$ $\mathbb{P}_{\mathbb{C}}^{n}$ is a cover of degree 4 with $R_{\text {planar }}(\varrho)=\emptyset$.

We cannot hope to show that each cover $\varrho: X \rightarrow \mathbb{P}_{\mathbb{C}}^{n}$ of degree 4 is a quadrisecant of some line bundle as for covers of degree 3 with $n \gg 0$ (see [Fj] or [La]).

Example 7.9. Let $Y, \mathcal{L}, \mathcal{E}, \mathcal{F}$ and $u, v, w$ be as in Example 4.6. $\mathcal{H}:=\check{\mathcal{F}} \otimes \mathcal{S}^{2} \mathcal{E}$ is globally generated; hence $X:=D_{0}\left(\Phi_{4}(\eta)\right) \subseteq \mathbb{P}$ is smooth for any general section $\eta \in H^{0}(Y, \mathcal{H})$. Up to a suitable linear transformation of the coordinates $u, v$ and $w, X \subseteq \mathbb{P}$ has global equations

$$
u^{2}+\alpha v w+\beta w^{2}=v^{2}+\gamma u w+\delta w^{2}=0,
$$

where $\alpha, \gamma \in H^{0}(Y, \mathcal{L}), \beta, \delta \in H^{0}\left(Y, \mathcal{L}^{2}\right)$. In particular, $X_{w}:=X \cap V_{+}(w)=\emptyset$; hence $\varrho:=\pi_{\mid X}: X \rightarrow Y$ is a cover of degree 4 with $X$ smooth for each value of $\operatorname{dim}(Y)$. On the other hand, it cannot be a quadrisecant of any line bundle, at least when $Y \cong \mathbb{P}_{\mathbb{C}}^{n}$ with $n \geq 4$.

\section{REFERENCES}

[A-K] Altman, A. Kleiman, S., Introduction to Grothendieck duality theory, L.N.M. 146, Springer, 1970. MR 43:224

[Ba] Barth, W., Counting singularities of quadratic forms on vector bundles, Vector bundles and differential equations (A. Hirschowitz, ed.), Birkhäuser, 1980, pp. 1-19. MR 82c: 14016

[Bea] Beauville, A., Variétés de Prym et jacobiennes intermédiaires, Ann. Scient. Éc. Norm. Sup. 10 (1977), pp. 309-391. MR 57:12532

[Cs] Casnati, G., Covers of algebraic varieties II. Covers of degree 5 and construction of surfaces, J. Algebraic Geom. 5 (1996), pp. 461-477. MR 97c:14015

[C-E] Casnati, G., Ekedahl, T., Covers of algebraic varieties I. A general structure theorem, covers of degree 3, 4 and Enriques surfaces, J. Algebraic Geom. 5 (1996), pp. 439-460. MR 97c:14014

[Ek] Ekedahl, T., letter to F. Catanese, 1990.

[Fj] Fujita, T., Triple covers by smooth manifolds, J. Fac. Sci. Univ. Tokyo, Sect. IA Math. 35 (1988), pp. 169-175. MR 89d:14019

[G-L] Gaffney, T., Lazarsfeld, R., On the ramification of branched coverings of $\mathbf{P}^{n}$, Invent. Math. 59 (1980), pp. 53-58. MR 81h:14012

[Ha] Hartshorne, R., Algebraic geometry, Springer, 1977. MR 57:3116

[Jo] Jouanolou, J.P., Théorèmes de Bertini et applications, Birkhäuser, 1983. MR 86b:13007

[La] Lazarsfeld, R., A Barth-type theorem for branched coverings of projective space, Math. Ann. 249 (1980), pp. 153-162. MR 81g:14007

[Mi] Miranda, R., Triple covers in algebraic geometry, Amer. J. Math. 107 (1985), pp. 11231158. MR 86k: 14008

[O-S-S] Okonek, C., Schneider, M., Spindler, H., Vector bundles on complex projective spaces, Birkhäuser, 1980. MR 81b:14001

[Re] Recillas, S., Jacobians of curves with $g_{4}^{1}$ 's are the Prym's of trigonal curves, Bol. Soc. Math. Mexicana 19 (1974), pp. 9-13. MR 58:666

[Sa] Sarkisov, V.G., On conic bundle structure, Math. USSR Izvestiya 20 (1982), pp. 355-390. MR 84h: 14047

[Ve] Verra, A., On Enriques surfaces as fourfold cover of $\mathbf{P}_{k}^{2}$, Math. Ann. 266 (1983), pp. 241-250. MR 85b:14051 
[Wa] Wall, C.T.C., Nets of conics, Math. Proc. Cambridge Phil. Soc. 81 (1977), pp. 351-364. MR 55:5652

[Wi] Wisniewski, J., On topological properties of some coverings. An addendum to a paper of Lanteri and Struppa, Canadian Jour. of Math. 44 (1992), pp. 206-214. MR 93a:14018

Dipartimento di Matematica Pura ed Applicata, Università degli Studi di Padova, via Belzoni 7, I-35131 Padova (Italy)

E-mail address: casnati@galileo.math.unipd.it 